\title{
High-Velocity Estimates and Inverse Scattering for Quantum N-Body Systems with Stark Effect*
}

\author{
Gerardo Daniel Valencia \\ Departamento de Física Matemática. \\ Instituto de Investigaciones en Matemáticas Aplicadas y en Sistemas. \\ Universidad Nacional Autónoma de México. \\ Apartado Postal 20-726. México, D.F. 01000. \\ e-mail: gvalenciamx@yahoo.com.mx \\ Ricardo Weder $\dagger$ \\ Projet POems, Domaine de Voluceau-Rocquencourt, Institut National de Recherche en \\ Informatique et en Automatique Paris-Rocquencourt, BP 105, 78153 Le Chesnay Cedex \\ France. \\ e-mail: weder@unam.mx
}

November 9, 2018

\begin{abstract}
In an $N$-body quantum system with a constant electric field, by inverse scattering, we uniquely reconstruct pair potentials, belonging to the optimal class of short-range potentials and longrange potentials, from the high-velocity limit of the Dollard scattering operator. We give a reconstruction formula with an error term.
\end{abstract}

\section{Introduction}

We study the direct and inverse scattering problems for an $N$-body quantum mechanical system in an $n \geq 2$ dimensional space under Stark effect, i.e. in a constant electric field, with interactions given by pair potentials (multiplication operators).

When we speak of scattering by a potential $V$, it is common that $V$ is classified as being short-range if the canonical wave operators $W_{ \pm}\left(H_{0}+V, H_{0}\right)$ exist, where $H_{0}$ is the unperturbed Hamiltonian. On the other hand, if they do not exist, we say that we have a long-range potential; in this case we have to modify the free evolution and thus, to define modified wave operators.

As it is well known, the Coulomb potential $V_{c}(x)=q /|x|$ is long-range when $H_{0}=-\triangle$. It is also well known, that $V_{c}$ is short-range in the case of the Stark effect, where $H_{0}=-\triangle-E \cdot x$, and $E$ is a constant electric field. More generally, potentials $V$ that decay at infinity as $V(x) \approx|x|^{-\gamma}, \gamma \leq 1$ are long-range when $H_{0}=-\triangle$ and on the contrary, when there is a constant electric field, they are short-range if $1 / 2<\gamma \leq 1$ and long-range if $0<\gamma \leq 1 / 2$.

\footnotetext{
*PACS Classification (2010): 03.65.Nk, 03.65.Ca, 03.65.Db, 03.65.Ta. Mathematics Subject Classification(2010): 81U40, 35P25, 35Q40, 35R30.

${ }^{\dagger}$ Fellow Sistema Nacional de Investigadores. Research partially supported by CONACYT under grant CB2008-I 99100.

${ }^{\ddagger}$ On leave of absence Departamento de Física Matemática. Instituto de Investigaciones en Matemáticas Aplicadas y en Sistemas. Universidad Nacional Autónoma de México. Apartado Postal 20-726. México, D.F. 01000.
} 
This feature of the Stark effect is particularly interesting in inverse scattering. For example, because it allows to prove that the Coulomb potential is uniquely determined by the scattering matrix, defined from canonical wave operators, without having to modify the free dynamics, as first proved in [39].

We denote by $m_{j} \in \mathbb{R}^{+}, q_{j} \in \mathbb{R}$ and $\tilde{\mathbf{x}}_{j} \in \mathbb{R}^{n}, j=1,2, \ldots, N$, respectively, the masses, the charges and the positions of the particles. The free Hamiltonian generates the free time evolution,

$$
\tilde{H}_{0}=\sum_{j=1}^{N}\left(2 m_{j}\right)^{-1} \tilde{\mathbf{p}}_{j}^{2}+\sum_{j=1}^{N} q_{j} \mathbf{E} \cdot \tilde{\mathbf{x}}_{j}, \quad \tilde{\mathbf{p}}_{j}=-i \nabla_{\tilde{\mathbf{x}}_{j}}
$$

where the electric field $\mathbf{E}=(-E, 0, \ldots, 0), E=|\mathbf{E}|>0$ is directed along minus the first coordinate direction.

We study the system in the center of mass frame and we separate off the motion of the center of mass

$$
H_{C M}=(2 M)^{-1}\left(\mathbf{P}_{C M}\right)^{2}+Q \mathbf{E} \cdot \mathbf{X}_{C M}
$$

where $M=\sum_{j=1}^{N} m_{j}$, is the total mass, $\mathbf{X}_{C M}=(1 / M) \sum_{j=1}^{N} m_{j} \tilde{\mathbf{x}}_{j}$, is the center of mass, $\mathbf{P}_{C M}=\sum_{j=1}^{N} \tilde{\mathbf{p}}_{j}$, is the momentum of the center of mass, $Q=\sum_{j=1}^{N} q_{j}$, is the total charge.

The free Hamiltonian in the center of mass frame is $H_{0}:=\tilde{H}_{0}-H_{C M}$,

$$
H_{0}=\sum_{j=1}^{N}\left(2 m_{j}\right)^{-1} \tilde{\mathbf{p}}_{j}^{2}-(2 M)^{-1}\left(\mathbf{P}_{C M}\right)^{2}+\sum_{j=1}^{N}\left(q_{j}-m_{j} Q / M\right) \mathbf{E} \cdot \tilde{\mathbf{x}}_{j} .
$$

$H_{0}$ is essentially self-adjoint in the space of Schwartz. We also denote by $H_{0}$ the unique self-adjoint extension.

In the center of mass frame the space of states is the Hilbert space, $\mathcal{H}$, represented in configuration space by wave functions $\phi$ in

$$
L^{2}(\mathbf{X}), \quad \mathbf{X}=\left\{\tilde{\mathbf{x}}=\left(\tilde{\mathbf{x}}_{1}, \ldots, \tilde{\mathbf{x}}_{N}\right) \mid \sum_{j=1}^{N} m_{j} \tilde{\mathbf{x}}_{j}=0\right\} \cong \mathbb{R}^{n(N-1)}
$$

with the measure induced on $\mathbf{X}$ by the following norm on $\mathbb{R}^{n N}:\left|\|\tilde{\mathbf{x}} \mid\|=\left[\sum_{j=1}^{N} m_{j} \tilde{\mathbf{x}}_{j}^{2}\right]^{1 / 2}\right.$. The space

$$
L^{2}(\hat{\mathbf{X}}), \quad \hat{\mathbf{X}}=\left\{\tilde{\mathbf{p}}=\left(\tilde{\mathbf{p}}_{1}, \ldots, \tilde{\mathbf{p}}_{N}\right) \mid \sum_{j=1}^{N} \tilde{\mathbf{p}}_{j}=0\right\} \cong \mathbb{R}^{n(N-1)}
$$

where $\hat{\mathbf{X}}$ is equipped with the dual metric induced by $\left[\sum_{j=1}^{N}\left(m_{j}\right)^{-1} \tilde{\mathbf{p}}_{j}^{2}\right]^{1 / 2}$ on $\mathbb{R}^{n N}$, is the set of momentum space wave functions $\hat{\phi}$. Fourier transform maps unitarily $L^{2}(\mathbf{X})$ onto $L^{2}(\hat{\mathbf{X}})$. The measures on $\mathbf{X}$ and $\hat{\mathbf{X}}$ are equivalent to Lebesgue measure. Given an (abstract) state $\Phi \in \mathcal{H}$ we use both its configuration or momentum space wave functions where appropriate.

As a general reference for multiparticle scattering see e.g. [35], where Jacobi coordinates are defined

$$
\xi_{j}:=\tilde{\mathbf{x}}_{j+1}-\left(\sum_{k=1}^{j} m_{k}\right)^{-1}\left(\sum_{k=1}^{j} m_{k} \tilde{\mathbf{x}}_{k}\right), \quad j=1, \ldots, N-1 .
$$


These coordinates are obtained by first changing variables from $\left(\tilde{\mathbf{x}}_{1}, \tilde{\mathbf{x}}_{2}\right)$ to $\xi_{1}=\tilde{\mathbf{x}}_{2}-\tilde{\mathbf{x}}_{1}$ and the center of mass of particles (1) and (2), $\tilde{\mathbf{R}}_{12}=\left(m_{1}+m_{2}\right)^{-1}\left(m_{1} \tilde{\mathbf{x}}_{1}+m_{2} \tilde{\mathbf{x}}_{2}\right)$. Then we change from $\left(\tilde{\mathbf{R}}_{12}, \tilde{\mathbf{x}}_{3}\right)$ to $\xi_{2}=\tilde{\mathbf{x}}_{3}-\tilde{\mathbf{R}}_{12}$ and the center of mass of particles (1), (2), and (3), and so on. In the end we obtain the Jacobi coordinates $\xi_{j}, 1 \leq j \leq N-1$, on $\mathbf{X}$ and the center of mass coordinate $\mathbf{X}_{C M}$. In these coordinates $H_{0}$ is expressed as

$$
H_{0}=\sum_{j=1}^{N-1}\left(\left(2 \nu_{j}\right)^{-1} \hat{\mathbf{p}}_{j}^{2}+q_{j}^{R} \mathbf{E} \cdot \xi_{j}\right), \quad \hat{\mathbf{p}}_{j}=-i \nabla_{\xi_{j}},
$$

where

$$
\begin{gathered}
\nu_{j}^{-1}=m_{j+1}^{-1}+\left(\sum_{k=1}^{j} m_{k}\right)^{-1}, \quad 1 \leq j \leq N-1, \\
q_{j}^{R}=\left(q_{j+1} M_{j}-m_{j+1} Q_{j}\right) /\left(m_{j+1}+M_{j}\right), \quad M_{j}=\sum_{k=1}^{j} m_{k}, \\
Q_{j}=\sum_{k=1}^{j} q_{k}, \quad 1 \leq j \leq N-1,
\end{gathered}
$$

$\nu_{j}$ and $q_{j}^{R}, 1 \leq j \leq N-1$, are, respectively, the reduced mass and the relative charge of the particle $(j+1)$ with respect to the masses and the charges of the first j particles. Formula (1.5) shows that the proof that $H_{0}$ is essentially self-adjoint in the space of Schwartz reduces to the one in the two-body case. The Jacobi coordinates above are based in the pair of particles $(1,2)$ in the sense that we have taken as the first coordinate $\xi_{1}=\tilde{\mathbf{x}}_{2}-\tilde{\mathbf{x}}_{1}$ the relative distance of the particles (1) and (2). Of course, we can base Jacobi coordinates in any pair of particles $(j, k), j, k=1,2, \ldots, N$.

In order to determine the potential for a given pair we number the particles in such a way that the given pair consists of particles one and two. By (1.5) we write

$$
H_{0}=\left[\left(2 \nu_{1}\right)^{-1} \hat{\mathbf{p}}_{1}^{2}+\frac{\left(q_{2} m_{1}-m_{2} q_{1}\right)}{m_{1}+m_{2}} \mathbf{E} \cdot \xi_{1}\right] \otimes I+I \otimes \hat{H}_{0}
$$

under the decomposition of $L^{2}(\mathbf{X})$ as

$$
L^{2}(\mathbf{X})=L^{2}\left(\mathbb{R}_{\xi_{1}}^{n}\right) \otimes\left[\otimes \prod_{j=2}^{N-1} L^{2}\left(\mathbb{R}_{\xi_{j}}^{n}\right)\right]
$$

where

$$
\hat{H}_{0}=\sum_{j=2}^{N-1}\left(\left(2 v_{j}\right)^{-1} \hat{\mathbf{p}}_{j}^{2}+q_{j}^{R} \mathbf{E} \cdot \xi_{j}\right) .
$$

This shows that if the relative charge of the pair $(1,2),\left(q_{2} m_{1}-m_{2} q_{1}\right) /\left(m_{1}+m_{2}\right)$, is different from zero the relative distance of the pair $(1,2)$ is accelerated by the electric field as in the two-body case. However, if the relative charge is zero both particles are accelerated in the same way by the electric field and the relative distance is not accelerated, and then, with respect to the pair $(1,2)$, the relative scattering is as in the case when the external constant electric field is zero. This shows that, for any given pair of particles, the inverse scattering problem has to be formulated as in the two-body case with no electric field if the relative charge of the pair is zero and, as in the two-body case with an electric field, if the relative charge of the pair is different from zero.

For any given pair of particles we construct as in Enss and Weder 19] appropriate states where all particles have high-velocity relative to each other in order to reconstruct the corresponding pair potential. 
For this purpose we first introduce some kinematical notation. We use a numbering of the particles such that the pair of interest consists of particles 1 and 2. As usual we take as one n-dimensional variable the relative distance $\mathbf{x}$ and momentum $\mathbf{p}$ of the chosen pair $(1,2)$.

$$
\left.\mathbf{x}=\xi_{1}:=\tilde{\mathbf{x}}_{2}-\tilde{\mathbf{x}}_{1}, \quad \mathbf{p}=\hat{\mathbf{p}}_{1}=-i \nabla_{\mathbf{x}}=\mu_{12}\left[\left(-i \nabla_{\tilde{\mathbf{x}}_{2}} / m_{2}\right)-\left(-i \nabla_{\tilde{\mathbf{x}}_{1}}\right) / m_{1}\right)\right]
$$

where $\mu_{12}$ is the reduced mass of the pair $(1,2), \mu_{12}=m_{1} m_{2} /\left(m_{1}+m_{2}\right)$. We also use the position $\mathbf{x}_{j}$ and the momentum $\mathbf{p}_{j}$ of the jth particle, $j=1, \ldots, N$, relative to the center of mass of the pair $(1,2)$,

$$
\begin{aligned}
& \mathbf{x}_{j}:=\tilde{\mathbf{x}}_{j}-\left(m_{1} \tilde{\mathbf{x}}_{1}+m_{2} \tilde{\mathbf{x}}_{2}\right) /\left(m_{1}+m_{2}\right), \quad j=1, \ldots, N \\
& \mathbf{p}_{j}=\mu_{j}\left(\tilde{\mathbf{p}}_{j} / m_{j}-\left(\tilde{\mathbf{p}}_{1}+\tilde{\mathbf{p}}_{2}\right) /\left(m_{1}+m_{2}\right)\right), \quad j=1, \ldots, N
\end{aligned}
$$

where $\mu_{j}$ is the reduced mass of the jth particle with respect to the center of mass of the pair $(1,2)$,

$$
\mu_{j}=m_{j}\left(m_{1}+m_{2}\right) /\left(m_{j}+m_{1}+m_{2}\right), \quad j=1, \ldots, N
$$

and $\tilde{\mathbf{p}}_{j}=-i \nabla_{\tilde{\mathbf{x}}_{j}}$ is the momentum relative to some origin (see (1.1)). Note that $\mathbf{x}$ is the first Jacobi coordinate $\xi_{1}, \mathbf{p}=\mu_{12}\left(\tilde{\mathbf{p}}_{2} / m_{2}-\tilde{\mathbf{p}}_{1} / m_{1}\right)$ and $\mathbf{p}_{j} / \mu_{j}$ are the relative velocities with respect the center of mass of the distinguished pair $(1,2) .\left\{\mathbf{x}, \mathbf{x}_{3}, \ldots \mathbf{x}_{N}\right\}$ and $\left\{\mathbf{p}, \mathbf{p}_{3}, \ldots \mathbf{p}_{N}\right\}$ are sets of $N-1$ independent ndimensional variables in the configuration and momentum space, respectively, relative to the center of mass frame.

Let $\Phi_{0} \in \mathcal{H}$ be an asymptotic configuration with the product wave function of the form in momentum space,

$$
\Phi_{0} \sim \hat{\phi}_{12}(\mathbf{p}) \hat{\phi}_{3}\left(\mathbf{p}_{3}, \ldots, \mathbf{p}_{N}\right)
$$

where $\hat{\phi}_{12} \in C_{0}^{\infty}\left(\mathbb{R}^{n}\right)$ varies while $\hat{\phi}_{3} \in C_{0}^{\infty}\left(\mathbb{R}^{n(N-2)}\right)$ is a fixed normalized function with support in $\left\{\left(\mathbf{p}_{3}, \ldots, \mathbf{p}_{N}\right):\left|\mathbf{p}_{j}\right|<\mu_{j}\right\}$; i.e., the particles 3 to $\mathrm{N}$ have speed smaller than one relative to the pair $(1,2)$. We take an $\eta>0$ such that $\hat{\phi}_{12} \in C_{0}^{\infty}\left(B_{\mu_{12} \eta}\right)$, where $B_{\mu_{12} \eta}$ denotes the open ball of center zero and radius $\mu_{12} \eta$ in $\mathbb{R}^{n}$.

The high-velocity state is defined as (see Enss and Weder [19])

$$
\Phi_{\mathbf{v}} \sim \hat{\phi}_{12}\left(\mathbf{p}-\mu_{12} \mathbf{v}\right) \hat{\phi}_{3}\left(\mathbf{p}_{3}-\mu_{3} \mathbf{v}_{3}, \ldots, \mathbf{p}_{N}-\mu_{N} \mathbf{v}_{N}\right)
$$

where $\mathbf{v}=v \hat{\mathbf{v}},|\hat{\mathbf{v}}|=1, \mathbf{v}_{j}=v^{2} \mathbf{d}_{j}$, with $\mathbf{d}_{j} \neq 0$, for $j=3, \ldots, N$ and where we assume that $\mathbf{d}_{j}-\mathbf{d}_{k} \neq 0$ for $j, k=3, \ldots N$. We, moreover, define $\mathbf{v}_{1}=-\mathbf{v} \mu_{12} / m_{1}, \mathbf{v}_{2}=\mathbf{v} \mu_{12} / m_{2}$.

We denote the relative velocities by

$$
\mathbf{v}_{j k}=\mathbf{v}_{k}-\mathbf{v}_{j}, \quad v_{j k}=\left|\mathbf{v}_{j k}\right|, \quad j, k=1, \ldots, N
$$

Then with $d_{j}=\left|\mathbf{d}_{j}\right|$

$$
\begin{aligned}
& \mathbf{v}_{1, j}=v^{2}\left(\mathbf{d}_{j}+\mu_{12} \hat{\mathbf{v}} /\left(m_{1} v\right)\right) \neq 0 \quad \text { if } v>\mu_{12} /\left(m_{1} d_{j}\right), j=3, \ldots, N, \\
& \mathbf{v}_{2, j}=v^{2}\left(\mathbf{d}_{j}-\mu_{12} \hat{\mathbf{v}} /\left(m_{2} v\right)\right) \neq 0 \quad \text { if } v>\mu_{12} /\left(m_{2} d_{j}\right), j=3, \ldots, N, \\
& \mathbf{v}_{j, k}=v^{2}\left(\mathbf{d}_{k}-\mathbf{d}_{j}\right) \neq 0 \quad j, k=3, \ldots, N .
\end{aligned}
$$

We denote $\hat{\mathbf{v}}_{j k}=\mathbf{v}_{j k} /\left|\mathbf{v}_{j k}\right|$. We assume for all pairs $(j, k)$ with $q_{j, k} \neq 0$ that $\left|\hat{\mathbf{v}}_{j k} \cdot \hat{\mathbf{E}}\right| \leq \delta$ for some $0 \leq \delta<1$. It follows that in our high-velocity states the relative average velocity of the pair $(1,2)$ is $v$ while all other particles travel with minimal velocity proportional to $v^{2}$ relative to each other as well as with respect to particles 1 and 2 .

The relative momentum of particles $j$ and $k$ is

$$
\mathbf{p}_{j k}=-i \nabla_{\left(\tilde{\mathbf{x}}_{k}-\tilde{\mathbf{x}}_{j}\right)}
$$


where in the derivative the positions of all other particles, as well as of the center of mass, are kept fixed. The relative velocity of the pair $(j, k)$ is

$$
\mathbf{p}_{j k} / \mu_{j k}=\tilde{\mathbf{p}}_{k} / m_{k}-\tilde{\mathbf{p}}_{j} / m_{j}=\mathbf{p}_{k} / \mu_{k}-\mathbf{p}_{j} / \mu_{j} .
$$

It follows from the definition that $\phi_{0} \in \mathcal{S}\left(\mathbb{R}^{n(N-1)}\right)$ and that

$$
\Phi_{\mathbf{v}}=e^{i \mu_{12} \mathbf{v} \cdot \mathbf{x}} \prod_{j=3}^{N} e^{i \mu_{j} \mathbf{v}_{j} \cdot \mathbf{x}_{j}} \Phi_{0} .
$$

Moreover, by (1.10)

$$
\left|\tilde{\mathbf{x}}_{k}-\tilde{\mathbf{x}}_{j}\right|=\left|\mathbf{x}_{k}-\mathbf{x}_{j}\right| \leq\left|\mathbf{x}_{k}\right|+\left|\mathbf{x}_{j}\right|, j, k=1, \cdots, N,\left|\mathbf{x}_{1}\right| \leq|\mathbf{x}|,\left|\mathbf{x}_{2}\right| \leq|\mathbf{x}| .
$$

Hence, we have good initial localization uniformly in $\mathbf{v}$,

$$
\left\|\left(1+\left|\tilde{\mathbf{x}}_{k}-\tilde{\mathbf{x}}_{j}\right|^{2}\right)^{2} \Phi_{\mathbf{v}}\right\| \leq C, \quad j, k=1, \ldots, N .
$$

Additionally, by (1.15) there are functions $f_{j k} \in C_{0}^{\infty}\left(B_{\mu_{j k}} \eta_{j k}\right)$ such that

$$
\Phi_{\mathbf{v}}=f_{j k}\left(\mathbf{p}_{j k}-\mu_{j k} \mathbf{v}_{j k}\right) \Phi_{\mathbf{v}}
$$

where $\mu_{j k}$ is the reduced mass of the pair $(j, k)$,

$$
\mu_{j k}=\frac{m_{j} m_{k}}{m_{j}+m_{k}}
$$

Furthermore, $\eta_{12}=\eta, \eta_{1 j}=2\left(1+\eta \mu_{12} / m_{1}\right), \eta_{2 j}=2\left(1+\eta \mu_{12} / m_{2}\right), j=3, \ldots, N$, and $\eta_{j k}=4$, for $j, k=3, \ldots, N$.

Note that by (1.10)

$$
\begin{gathered}
\mathbf{x}=\tilde{\mathbf{x}}_{2}-\tilde{\mathbf{x}}_{1}=i \frac{\partial}{\partial \mathbf{p}}, \quad \tilde{\mathbf{x}}_{k}-\tilde{\mathbf{x}}_{j}=i \frac{\partial}{\partial \mathbf{p}_{k}}-i \frac{\partial}{\partial \mathbf{p}_{j}}, \quad j, k=3, \ldots, N, \\
\tilde{\mathbf{x}}_{k}-\tilde{\mathbf{x}}_{1}=i \frac{\partial}{\partial \mathbf{p}_{k}}+\frac{\mu_{12}}{m_{1}} i \frac{\partial}{\partial \mathbf{p}}, \quad \tilde{\mathbf{x}}_{k}-\tilde{\mathbf{x}}_{2}=i \frac{\partial}{\partial \mathbf{p}_{k}}-\frac{\mu_{12}}{m_{2}} i \frac{\partial}{\partial \mathbf{p}}, \quad k=3, \ldots, N .
\end{gathered}
$$

As in Enss and Weder [19] and Weder [39, (1.18), (1.19), (1.21) and (1.22) allow us to reduce the proofs in the N-body case to the ones for two bodies. We introduce below an appropriate class of potentials where $D^{\beta}, D^{\alpha_{0}}$ denotes the derivative with the usual multi-index notation.

DEFINITION 1.1. We denote by $\mathcal{V}_{0}$ the class of real-valued potentials, $V^{0}(\mathbf{x})$, defined on $\mathbb{R}^{n}$ with values in $\mathbb{R}$ such that $V^{0}(\mathbf{x})=V^{0, v s}(\mathbf{x})+V^{0, l}(\mathbf{x})$ with $V^{0, v s}(\mathbf{x}) \in \mathcal{V}_{0, v s}, V^{0, l}(\mathbf{x}) \in \mathcal{V}_{0, l}$, where $\mathcal{V}_{0, \text { vs }}$ is the class of real-valued potentials, $V^{0, v s}$, that are relatively bounded with respect to the Laplacian with relative bound zero and

$$
\int_{0}^{\infty} d R\left\|V^{0, v s}(\mathbf{x})(-\Delta+I)^{-1} F(|\mathbf{x}| \geq R)\right\|<\infty .
$$

$\mathcal{V}_{0, l}$ is the class of real-valued potentials $V^{0, l}$ that satisfy $V^{0, l}(\mathbf{x}) \in C_{\infty}^{1}\left(\mathbb{R}^{n}\right)$, the space of all continuously differentiable functions that tend to zero at infinity, and that

$$
\left|D^{\beta} V^{0, l}(\mathbf{x})\right| \leq C(1+|\mathbf{x}|)^{-\gamma_{1}}, \quad|\beta|=1, \gamma_{1}>3 / 2,
$$

where without loss of generality we assume that $\gamma_{1} \leq 2$, otherwise $V^{0, l}$ would be of short range. 
Let $\epsilon_{0}$ satisfy: $0<\epsilon_{0}<\gamma_{1}-\frac{3}{2}$. After Hörmander [24], we can write, without loss of generality that, for all $V^{0}(\mathbf{x}) \in \mathcal{V}_{0}, V^{0}(\mathbf{x})=V^{0, v s}(\mathbf{x})+V^{0, l}(\mathbf{x})$ with $V^{0, v s} \in \mathcal{V}_{0, v s}, V^{0, l} \in C^{4}\left(\mathbb{R}^{n}\right)$ and

$$
\left|D^{\alpha_{0}} V^{0, l}(\mathbf{x})\right| \leq C(1+|\mathbf{x}|)^{-1-\left|\alpha_{0}\right|\left(\epsilon_{0}+1 / 2\right)}, \text { for } 2 \leq\left|\alpha_{0}\right| \leq 4
$$

The more intuitive condition

$$
\int_{0}^{\infty} d R\left\|F(|\mathbf{x}| \geq R) V^{0, v s}(\mathbf{x})(-\Delta+I)^{-1}\right\|<\infty,
$$

by Reed and Simon [35], is equivalent to the decay property (1.23).

DEFINITION 1.2. [2]. We denote by $\mathcal{V}_{E}$ the class of potentials, $V^{E}(\mathbf{x})$, defined on $\mathbb{R}^{n}$ with values in $\mathbb{R}$ such that $V^{E}(\mathbf{x})=V^{E, v s}(\mathbf{x})+V^{E, s}(\mathbf{x})+V^{E, l}(\mathbf{x})$ with $V^{E, v s}(\mathbf{x}) \in \mathcal{V}_{E, v s}, V^{E, s}(\mathbf{x}) \in \mathcal{V}_{E, s}$, and $V^{E, l}(\mathbf{x}) \in \mathcal{V}_{E, l}$, where $\mathcal{V}_{E, \text { vs }}$ is the class of real-valued potentials, $V^{E, v s}$, that satisfy $V^{E, v s}=V_{1}^{E, v s}+V_{2}^{E, v s}$ with $\left(1+\left|x_{1}\right|\right) V_{1}^{E, v s}$ relatively bounded with respect to the Laplacian with relative bound zero and $V_{2}^{E, v s}$ bounded and that

$$
\int_{0}^{\infty} d R\left\|V^{E, v s}(\mathbf{x})(-\Delta+I)^{-1} F(|\mathbf{x}| \geq R)\right\|<\infty .
$$

$\mathcal{V}_{E, s}$ is the class of real-valued potentials $V^{E, s}$ that satisfy $V^{E, s}(\mathbf{x}) \in C^{1}\left(\mathbb{R}^{n}\right)$ and that

$$
\begin{aligned}
\left|V^{E, s}(\mathbf{x})\right| & \leq C(1+|\mathbf{x}|)^{-\gamma}, \\
\left|D^{\beta} V^{E, s}(\mathbf{x})\right| & \leq C(1+|\mathbf{x}|)^{-1-\alpha}, \quad|\beta|=1,
\end{aligned}
$$

with some $1 / 2<\alpha \leq \gamma \leq 1$. $\mathcal{V}_{E, l}$ is the class of real-valued potentials $V^{E, l}$ that satisfy $V^{E, l}(\mathbf{x}) \in C^{2}\left(\mathbb{R}^{n}\right)$ and that

$$
\left|D^{\beta} V^{E, l}(\mathbf{x})\right| \leq C(1+|\mathbf{x}|)^{-\gamma_{D}-\mu|\beta|}, \quad|\beta| \leq 2,
$$

with $0<\gamma_{D} \leq 1 / 2$ and $1-\gamma_{D}<\mu \leq 1$.

The class of potentials $\mathcal{V}_{E}$ in Definition 1.2 is the same as in Adachi and Maehara 2]. Again we can assume, without loss of generality by [24, that for all $V^{E}(\mathbf{x}) \in \mathcal{V}_{E}, V^{E}(\mathbf{x})=V^{E, v s}(\mathbf{x})+V^{E, s}(\mathbf{x})+V^{E, l}(\mathbf{x})$ with $V^{0, v s} \in \mathcal{V}_{0, v s}, V^{E, s}(\mathbf{x}) \in \mathcal{V}_{E, s}, V^{E, l} \in C^{4}\left(\mathbb{R}^{n}\right)$ with $V^{E, l}$ satisfying (1.29) and

$$
\left|D^{\beta} V^{E, l}(\mathbf{x})\right| \leq C(1+|\mathbf{x}|)^{-\gamma_{D}-\mu(2+|\beta|) / 2}, \quad 3 \leq|\beta| \leq 4 .
$$

We call the potentials $V^{0, v s}$ and $V^{E, v s}$ very short-range, the potential $V^{E, s}$ short-range and the potentials $V^{0, l}$ and $V^{E, l}$ long-range.

For a particle with mass $m$ and charge $q$, there is a formula for the free time evolution, it was proven simultaneously by Avron and Herbst [7] and by Veselić and Weidmann [38. There is also a generalization for the time-dependent case considered by Kitada and Yajima 30],

$$
e^{-i t\left(\mathbf{p}^{2} /(2 m)-q E \mathbf{x}_{1}\right)}=e^{i q E \mathbf{x}_{1} t} e^{-i t^{3} q^{2} E^{2} /(6 m)} e^{-i \mathbf{p}_{1} q E t^{2} /(2 m)} e^{-i t \mathbf{p}^{2} /(2 m)} .
$$

We will also make frequent use of the following relations that are obtained under translation in configuration or momentum space generated by $\mathbf{x}$ or $\mathbf{p}$, respectively,

$$
\begin{aligned}
e^{i \mathbf{p} \cdot \mathbf{v} t} f(\mathbf{x}) e^{-i \mathbf{p} \cdot \mathbf{v} t} & =f(\mathbf{x}+\mathbf{v} t), \\
e^{-i m \mathbf{v} \cdot \mathbf{x}} f(\mathbf{p}) e^{i m \mathbf{v} \cdot \mathbf{x}} & =f(\mathbf{p}+m \mathbf{v}),
\end{aligned}
$$

for any measurable and bounded function $\mathrm{f}$. In particular, (1.33) implies that

$$
e^{-i m \mathbf{v} \cdot \mathbf{x}} e^{-i t \mathbf{p}^{2} /(2 m)} e^{i m \mathbf{v} \cdot \mathbf{x}}=e^{-i \mathbf{p} \cdot \mathbf{v} t} e^{-i t \mathbf{p}^{2} /(2 m)} e^{-i m v^{2} t / 2}
$$


where $v=|\mathbf{v}|$. Since $e^{i t \mathbf{p}^{2} /(2 m)} \mathbf{x} e^{-i t \mathbf{p}^{2} /(2 m)}=\mathbf{x}+t \mathbf{p} / m$ and functional calculus,

$$
e^{i t \mathbf{p}^{2} /(2 m)} f(\mathbf{x}) e^{-i t \mathbf{p}^{2} /(2 m)}=f(\mathbf{x}+t \mathbf{p} / m) .
$$

We denote by $\mathbf{e}_{1}=(1,0, \ldots, 0)$ the unit vector along the $x_{1}$ direction and $\hat{\mathbf{E}}=\mathbf{E} /|\mathbf{E}|$. We designate by $q_{j k}=\left(q_{k} m_{j}-q_{j} m_{k}\right) /\left(m_{j}+m_{k}\right)$ the relative charge of the pair $(j, k)$ and we denote by $\sum_{j<k}^{0}$ and $\sum_{j<k}^{E}$, respectively, the sum over all indices, $j<k, j, k=1, \ldots, N$, with $q_{j k}=0$, and $q_{j k} \neq 0$.

We assume that the potential of the N-body system is a multiplication operator that is a sum of pair potentials,

$$
V=\sum_{j<k}^{0} V_{j k}^{0}\left(\tilde{\mathbf{x}}_{k}-\tilde{\mathbf{x}}_{j}\right)+\sum_{j<k}^{E} V_{j k}^{E}\left(\tilde{\mathbf{x}}_{k}-\tilde{\mathbf{x}}_{j}\right),
$$

with $V_{j k}^{0} \in \mathcal{V}_{0}$ (see Definition 1.1), and $V_{j k}^{E} \in \mathcal{V}_{E}$ (see Definition 1.2). By using a decomposition of $H_{0}$ as in (1.7) for each pair $(j, k)$ we see that each of the pair potentials $V_{j k}^{0}$ and $V_{j k}^{E}$ are relatively bounded with respect to $H_{0}$ with relative bound zero. Note that for a given pair the corresponding pair potential belongs to $\mathcal{V}_{0}$ if the relative charge of the pair is zero and that it belongs to $\mathcal{V}_{E}$ if the relative charge is different from zero. Then $\mathrm{V}$ is relatively bounded with respect to $H_{0}$ with relative bound zero and the interacting Hamiltonian,

$$
H=H_{0}+V
$$

is self-adjoint on $D(H)=D\left(H_{0}\right)$.

It is convenient to split the potential into the very short-, short- and long-range potentials. For this purpose we define

$$
\begin{gathered}
\mathcal{V}_{V S R}=\left\{V^{V S}=\sum_{j<k}^{0} V_{j k}^{0, v s}\left(\tilde{\mathbf{x}}_{k}-\tilde{\mathbf{x}}_{j}\right)+\sum_{j<k}^{E} V_{j k}^{E, v s}\left(\tilde{\mathbf{x}}_{k}-\tilde{\mathbf{x}}_{j}\right) \mid V_{j k}^{0, v s} \in \mathcal{V}_{0, v s}, V_{j k}^{E, v s} \in \mathcal{V}_{E, v s}\right\}, \\
\mathcal{V}_{S R}=\left\{V^{S}=\sum_{j<k}^{E} V_{j k}^{E, s}\left(\tilde{\mathbf{x}}_{k}-\tilde{\mathbf{x}}_{j}\right) \mid V_{j k}^{E, s} \in \mathcal{V}_{E, s}\right\} \\
\mathcal{V}_{L R}=\left\{V^{L}=\sum_{j<k}^{0} V_{j k}^{0, l}\left(\tilde{\mathbf{x}}_{k}-\tilde{\mathbf{x}}_{j}\right)+\sum_{j<k}^{E} V_{j k}^{E, l}\left(\tilde{\mathbf{x}}_{k}-\tilde{\mathbf{x}}_{j}\right) \mid V_{j k}^{0, l} \in \mathcal{V}_{0, l}, V_{j k}^{E, l} \in \mathcal{V}_{E, l}\right\}
\end{gathered}
$$

Then

$$
V=V^{V S}+V^{S}+V^{L}, \quad H=H_{0}+V=H_{0}+V^{V S}+V^{S}+V^{L} .
$$

Let $S^{D}=S^{D}\left(V^{L} ; V^{V S}+V^{S}\right)$ be the Dollard modified scattering operator defined in equation (2.10) below.

Our main results are the reconstruction formulae given in Theorems 2.8 and 2.10 that we prove in Section [I] The uniqueness result given in Theorem 1.3 follows from Theorem 2.8 .

THEOREM 1.3. Let $\gamma_{1}$ be as in Definition 1.1 and, $\gamma_{D}$ and $\mu$ as in Definition 1.2. If there are two pairs $1 \leq j<k \leq N, 1 \leq j^{\prime}<k^{\prime} \leq N$, with $q_{j k} \neq 0$ and $q_{j^{\prime} k^{\prime}}=0$ we assume that $\gamma_{1}>3-4\left(\gamma_{D}+\mu\right) / 3$. Then,

1. Suppose that $V^{i}=V^{V S, i}+V^{S, i}+V^{L, i} \in \mathcal{V}_{V S R}+\mathcal{V}_{S R}+\mathcal{V}_{L R}, i=1,2$, and that $S^{D}\left(V^{L, 1} ; V^{V S, 1}+\right.$ $\left.V^{S, 1}\right)=S^{D}\left(V^{L, 2} ; V^{V S, 2}+V^{S, 2}\right)$. Then, $V^{1}=V^{2}$.

2. Furthermore, it is possible to uniquely reconstruct the total potential $V$ from any Dollard scattering operator $S^{D}$. 
REMARK 1.4. Note that in item 1 of Theorem 1.3 it is enough to assume that the high-velocity limits of $S^{D}\left(V^{L, 1} ; V^{V S, 1}+V^{S, 1}\right)$ and $S^{D}\left(V^{L, 2} ; V^{V S, 2}+V^{S, 2}\right)$ are the same. Furthermore, we prove item 2 of Theorem 1.3 giving a method for the unique reconstruction of $V$ from the high-velocity limit of any Dollard scattering operator. See the reconstruction formulae (2.44), (2.75) and the proof of Theorem 1.3 ,

REMARK 1.5. For a given $V^{L} \in \mathcal{V}_{L R}$ let us define, as in [2] and [39], the scattering map $S_{1}:=S^{D}\left(V^{L} ; \cdot\right)$, $S_{1}(Q)=S^{D}\left(V^{L} ; Q\right), Q \in \mathcal{V}_{V S R}+\mathcal{V}_{S R}$, an operator from $\mathcal{V}_{V S R}+\mathcal{V}_{S R}$ into the Banach space $\mathcal{L}(\mathcal{H})$ of all bounded operators in $\mathcal{H}$. Clearly, Theorem 1.3 implies that $S_{1}=S^{D}\left(V^{L} ; \cdot\right)$ is injective.

REMARK 1.6. For a given $V^{L} \in \mathcal{V}_{L R}$ and a given $V^{S} \in \mathcal{V}_{S R}$ we define the scattering map $S_{2}:=$ $S^{D}\left(V^{L} ; \cdot+V^{S}\right), S_{2}\left(V^{V S}\right)=S_{2}\left(V^{L} ; V^{V S}+V^{S}\right)$, an operator from $\mathcal{V}_{V S R}$ into $\mathcal{L}(\mathcal{H})$. It is immediate that Theorem 1.3 implies that $S_{2}=S^{D}\left(V^{L} ; \cdot+V^{S}\right)$ is injective. However, as we show in Remark 2.11 this result can also be proven using the reconstruction formula (2.75) given in Theorem 2.10, that is simpler than the formula (2.44) in Theorem 2.8, because in (2.75) it is not necessary to take the commutator of $S^{D}$ with a component of the momentum operator. This is important in applications where the tail at infinity of the potential is already known and one wishes to uniquely reconstruct $V^{V S}$ assuming that $V^{S}$ and $V^{L}$ are known.

REMARK 1.7. Under the Stark effect, for a pair potential where the relative charge is not zero, the short-range decay rate at infinity of this potential depends on $\gamma$ given in our equation (1.27). Theorem 1.3 is proved by the first time by Weder [39, where he considers $\gamma>3 / 4$ and N-Body pair potentials which are short-range if the corresponding relative charge is not zero and long-range if the corresponding relative charge is zero. Then, for two body short-range potentials, Nicoleau [33] proves this Theorem with $\gamma>1 / 2$, the dimension of the space $n \geq 3$ and the regularity and decay of the potential:

$$
V: \quad\left|\partial_{x}^{\beta} V(x)\right| \leq C_{\beta}(1+|x|)^{-\gamma-|\beta|},
$$

for all multi-index $\beta$. Later, in the two-body case, Adachi and Maehara [2] improve the results of Nicoleau [33] because, besides $\gamma>1 / 2$, they relax the conditions on the derivatives on the potential and use dimension $n \geq 2$. Furthermore, Adachi and Maehara [2] consider long-range potentials whereas Nicoleau [33] does not.

We improve the N-body results of Weder [39]. Our potential $V$ is given, by

$$
V=\sum_{j<k}^{0}\left(V_{j k}^{0, v s}\left(\tilde{\mathbf{x}}_{k}-\tilde{\mathbf{x}}_{j}\right)+V_{j k}^{0, l}\left(\tilde{\mathbf{x}}_{k}-\tilde{\mathbf{x}}_{j}\right)\right)+\sum_{j<k}^{E}\left(V_{j k}^{E, v s}\left(\tilde{\mathbf{x}}_{k}-\tilde{\mathbf{x}}_{j}\right)+V_{j k}^{E, s}\left(\tilde{\mathbf{x}}_{k}-\tilde{\mathbf{x}}_{j}\right)+V_{j k}^{E, l}\left(\tilde{\mathbf{x}}_{k}-\tilde{\mathbf{x}}_{j}\right)\right)
$$

where, for all $1 \leq j<k \leq N, V_{j k}^{0, v s} \in \mathcal{V}_{0, v s}, V_{j k}^{0, l} \in \mathcal{V}_{0, l}, V_{j k}^{E, v s} \in \mathcal{V}_{E, v s}, V_{j k}^{E, s} \in \mathcal{V}_{E, s}, V_{j k}^{E, l} \in \mathcal{V}_{E, l}$. Our potential $V_{j k}^{E, l}$ has no counterpart in [39], i.e. potentials that are long-range with respect to the Stark effect, when the relative charge $q_{j k} \neq 0$, are not allowed in [39] whereas, here, we do. This is our first improvement over [39]. Secondly, in equation (1.4) of [39] $\gamma>3 / 4$ and in our equation (1.27) we have $\gamma>1 / 2$, thus we improve the results of [39] because our potential $V_{j k}^{E, s}$ is allowed to have the optimal short-range decay rate at infinity.

We give a reconstruction formula with an error term that goes to zero as an inverse power of the velocity, that depends on the decay rate of the potentials, see Theorems 2.8 and 2.10 . If we only assume (1.26), our results coincide with those of Adachi and Maehara [2], in the case $N=2$ and $q_{12} \neq 0$. If, instead of (1.26), we assume (2.21), we give a sharper error term than theirs. In this sense, we can say that we obtain a new result, even in the two body case.

In this paper, we prove Theorem 1.3 by extending to the N-body case the results obtained, in the two-body case, by Adachi and Maehara [2] using the the findings published in 1993 [17, 1994 [18, 1995 [19] by V. Enss and R. Weder where a new time-dependent method was developed. Here, physical propagation properties of finite energy wave functions are used to estimate the high-velocity behavior of solutions of the Schrödinger equation and solve inverse scattering problems in quantum mechanics. It is intuitive from the point of view 
of the physics related to the problem. Contrary to the stationary approach, this method can be applied to study non-linear equations [36, 41, 47, 49,51,53]. Lately, this time-dependent approach has been exploited to study: Hamiltonians with electric and magnetic fields [4,6, 29, N-body systems [18, 20, 39, 40, the Stark effect [1,2,33,34,39], the Aharanov-Bohm effect [8, 10,32,48,54,55], time-dependent potentials [34, 40, Dirac equation [15, 25, 28, Klein-Gordon equation [15, 16, 44, 49, mass and charge of black holes [11,12], amongst others.

\section{Reconstruction Formulae}

Let us define

$$
V_{j k}^{v s}=\left\{\begin{array}{ll}
V_{j k}^{0, v s}, & \text { if } q_{j k}=0, \\
V_{j k}^{E, v s}, & \text { if } q_{j k} \neq 0,
\end{array} \quad V_{j k}^{s}=\left\{\begin{array}{ll}
0, & \text { if } q_{j k}=0, \\
V_{j k}^{E, s}, & \text { if } q_{j k} \neq 0,
\end{array} \quad V_{j k}^{l}= \begin{cases}V_{j k}^{0, l}, & \text { if } q_{j k}=0 \\
V_{j k}^{E, l}, & \text { if } q_{j k} \neq 0 .\end{cases}\right.\right.
$$

where $V_{j k}^{0, v s}$ and $V_{j k}^{0, l}$ are defined in Definition 1.1. and $V_{j k}^{E, v s}, V_{j k}^{E, s}$ and $V_{j k}^{E, l}$ are defined in Definition 1.2. Moreover, the decays of $V_{j k}^{0, l}$ and $V_{j k}^{E, l}$ are to be taken as in (1.25), (1.29) and (1.30), respectively.

We introduce the Graf-type modifier (2.2) Graf [21] and Zorbas [56], to define auxiliary wave operators, whose existence and completeness were proven in the $\mathrm{N}$ body case for long-range Stark Hamiltonians by Adachi and Tamura [3. We note that the $\mathbf{v}$ dependence of the Graf-type modifier (2.2) is first introduced in Adachi and Maehara [2] by taking into account the $\mathbf{v}$ dependence of $\Phi_{\mathbf{v}}$. In Graf [21] and Zorbas [56], $\mathbf{v}$ is taken 0 in the definiton of the Graf-type modified wave operators. We define the Graf-type modifier [2], 21], [56] and the Dollard-type modifier by (2.2), and (2.3), respectively

$$
\begin{aligned}
\tilde{U}_{G, v}(t) & =\exp \left(-i \sum_{j<k}^{E} \int_{0}^{t} d s V_{j k}^{E, s}\left(\mathbf{v}_{j k} s+\mathbf{e}_{1} q_{j k} E s^{2} /\left(2 \mu_{j k}\right)\right)\right), \\
\tilde{U}_{D}(t) & =\exp \left(-i \sum_{j<k} \int_{0}^{t} d s V_{j k}^{l}\left(s \mathbf{p}_{j k} / \mu_{j k}+\mathbf{e}_{1} q_{j k} E s^{2} /\left(2 \mu_{j k}\right)\right)\right) .
\end{aligned}
$$

For completeness we mention, for the short-range case, the modified Graf propagator, the modified Graf wave operators [21], [56] and the wave operators for the free channel which are defined, respectively, by (2.4) (2.5) and (2.6):

$$
\begin{aligned}
U^{G, v}(t) & =e^{-i t H_{0}} \tilde{U}_{G, v}(t), \\
\Omega_{ \pm}^{G, v} & =\mathrm{s}-\lim _{t \rightarrow \pm \infty} e^{i t H} U^{G, v}(t), \\
W_{ \pm} & =\mathrm{s}-\lim _{t \rightarrow \pm \infty} e^{i t H} e^{-i t H_{0}}
\end{aligned}
$$

The modified Dollard-Graf propagator, the modified Dollard-Graf wave operators [21], [56] and the modified Dollard wave operators for the free channel are defined, respectively, by (2.7) (2.8) and (2.9):

$$
\begin{aligned}
U^{D, G, v}(t) & =e^{-i t H_{0}} \tilde{U}_{D}(t) \tilde{U}_{G, v}(t) \\
\Omega_{ \pm}^{D, G, v} & =s-\lim _{t \rightarrow \pm \infty} e^{i t H} U^{D, G, v}(t) \\
W_{ \pm}^{D} & =s-\lim _{t \rightarrow \pm \infty} e^{i t H} e^{-i t H_{0}} \tilde{U}_{D}(t) .
\end{aligned}
$$

Tamura proved the existence of the $W_{ \pm}$for short range N-Body Stark systems [37, Korotyaev [31] did it for the case $\mathrm{N}=3$. Adachi and Tamura [3], and, Herbst, Møller and Skibsted [23] proved the existence of $W_{ \pm}^{D}$ given by (2.9) for the N-Body long-range case. Actually, the existence of the $W_{ \pm}^{D}$ and $W_{ \pm}$also follows 
from our estimates. We give the simple proof of the existence of $\Omega_{ \pm}^{D, G, v}$ and $\Omega_{ \pm}^{G, v}$ in Proposition 2.6 The Dollard scattering operator $S^{D}$ from the free channel to the free channel is defined as

$$
S^{D}=S^{D}\left(V^{L} ; V^{V S}+V^{S}\right):=\left(W_{+}^{D}\right)^{*} W_{-}^{D},
$$

$S^{D}$ is not unique because there is more than one short- and long-range splitting of the potential. We also mention the scattering operator $S$ from the free channel to the free channel defined for the short-range case as

$$
S=\left(W_{+}\right)^{*} W_{-} .
$$

Proposition 2.1, below, shall be frequently used in this text. Its proof is given in the Proposition 2.10 in Enss [14.

PROPOSITION 2.1. For any $f \in C_{0}^{\infty}\left(\mathbb{R}^{n}\right)$ with supp $f \subset B_{m \eta_{0}}$, for some $m, \eta_{0}>0$ and any $l=1,2,3, \ldots$ there is a constant $C_{l}$ such that the following estimate is true:

$$
\left\|F\left(\mathbf{x} \in \mathcal{M}^{\prime}\right) e^{-i t \mathbf{p}^{2} /(2 m)} f(\mathbf{p}-m \mathbf{v}) F(\mathbf{x} \in \mathcal{M})\right\| \leq C_{l}(1+r+|t|)^{-l},
$$

for every $\mathbf{v} \in \mathbb{R}^{n}, t \in \mathbb{R}$ and any measurable sets $\mathcal{M}^{\prime}, \mathcal{M}$ such that $r:=\operatorname{dist}\left(\mathcal{M}^{\prime}, \mathcal{M}+\mathbf{v} t\right)-\eta_{0}|t|>0$.

To treat the case whether or not the relative charge $q_{j k}$ is zero, we define

$$
\delta_{j k}:= \begin{cases}\delta, & \text { if } q_{j k} \neq 0 \\ 0, & \text { if } q_{j k}=0\end{cases}
$$

where $\delta$ is such that $\left|\hat{\mathbf{v}}_{j k} \cdot \hat{\mathbf{E}}\right| \leq \delta<1$, for all integers $1 \leq j<k \leq N$ with $q_{j k} \neq 0$.

A cornerstone throughout this work is the existence of $0<\delta_{1}, \delta_{2} \leq 1$ such that

$$
\left|\mathbf{v} t+\mathbf{e}_{1} q_{12} E t^{2} /\left(2 \mu_{12}\right)\right| \geq \sqrt{\delta_{1}|v t|^{2}+\delta_{2}\left(q_{12} E /\left(2 \mu_{12}\right)\right)^{2} t^{4}} \geq \sqrt{\delta_{1}}|v t| .
$$

When $q_{12}=0$, we can take $\delta_{1}=\delta_{2}=1$, and if $q_{12} \neq 0$, we use $\delta_{1}=\delta_{2}=1-\delta$, Moreover, if $0 \leq \tilde{\sigma} \leq 1, q_{12} \neq$ $0,|\mathbf{p}| \leq \mu_{12} \eta$, and $\eta / v<\sqrt{1-\delta} / 4$, from a simple computation, there exist two positive constants $c_{1}$ and $c_{2}$ such that

$$
\begin{gathered}
\left|t \mathbf{p} / \mu_{12}+\mathbf{v} t+\mathbf{e}_{1} q_{12} E t^{2} /\left(2 \mu_{12}\right)\right| \geq c_{1}|v t|, \\
\left|t \mathbf{p} / \mu_{12}+\mathbf{v} t+\mathbf{e}_{1} q_{12} E t^{2} /\left(2 \mu_{12}\right)\right| \geq c_{2} t^{2}, \\
\left|t \mathbf{p} / \mu_{12}+\mathbf{v} t+\mathbf{e}_{1} q_{12} E t^{2} /\left(2 \mu_{12}\right)\right| \geq c_{1}^{\tilde{\sigma}} c_{2}^{1-\tilde{\sigma}}|v t|^{\tilde{\sigma}} t^{2(1-\tilde{\sigma})} .
\end{gathered}
$$

For any pair $(j, k)$, we establish three conditions: $\zeta_{j k}^{a}$ as " $\gamma_{1}<2$ and there is, at least, one pair $\left(j^{\prime}, k^{\prime}\right)$ with $q_{j^{\prime} k^{\prime}}=0, V_{j^{\prime}, k^{\prime}}^{l} \neq 0$, and either $j^{\prime}=j$ or $j^{\prime}=k$ or $k^{\prime}=j$ or $k^{\prime}=k$ or $j^{\prime}+j=3$ ", $\zeta_{j k}^{b}$ as " $\gamma_{1}=2$ and there is, at least, one pair $\left(j^{\prime}, k^{\prime}\right)$ with $q_{j^{\prime} k^{\prime}}=0, V_{j^{\prime}, k^{\prime}}^{l} \neq 0$, and either $j^{\prime}=j$ or $j^{\prime}=k$ or $k^{\prime}=$ $j$ or $k^{\prime}=k$ or $j^{\prime}+j=3$ ", and $\zeta_{j k}^{c}$ as "there is no pair $\left(j^{\prime}, k^{\prime}\right)$ with $q_{j^{\prime} k^{\prime}}=0, V_{j^{\prime}, k^{\prime}}^{l} \neq 0$, and either $j^{\prime}=j$ or $j^{\prime}=k$ or $k^{\prime}=j$ or $k^{\prime}=k$ or $j^{\prime}+j=3$ ". We define the following constant, for any $\epsilon>0$ :

$$
\theta_{j k}:= \begin{cases}2-\gamma_{1}, & \text { if } \zeta_{j k}^{a}, \\ \epsilon, & \text { if } \zeta_{j k}^{b}, \\ 0, & \text { if } \zeta_{j k}^{c} .\end{cases}
$$

LEMMA 2.2. Let $\tilde{U}_{D}(t)$ be given as in (2.3). Then there exists a constant $C$, such that for all $t \in \mathbb{R}$, for every $\mathbf{v}_{j k} \in \mathbb{R}^{n}$, as in (1.14), with $v_{j k} \geq 4 \eta_{j k} / \sqrt{1-\delta_{j k}}$ and $v=v_{12}$, for all $f_{j k} \in C_{0}^{\infty}\left(B_{\mu_{j k} \eta_{j k}}\right)$, for all integers $1 \leq j<k \leq N$, for all $\kappa>0$ and $0<\tilde{\epsilon}<\min \left\{4 \epsilon_{0}, 2 \gamma_{D}+5 \mu+\epsilon_{0}-5 / 2,2 \gamma_{D}+6 \mu-3\right\}$, one has that 


$$
\begin{aligned}
A_{j k} & :=\left\|\left(\tilde{\mathbf{x}}_{k}-\tilde{\mathbf{x}}_{j}\right) \tilde{U}_{D}(t) \prod_{j^{\prime}<k^{\prime}} f_{j^{\prime} k^{\prime}}\left(\mathbf{p}_{j^{\prime} k^{\prime}}-\mu_{j^{\prime} k^{\prime}} \mathbf{v}_{j^{\prime} k^{\prime}}\right)\left(1+\left|\tilde{\mathbf{x}}_{k}-\tilde{\mathbf{x}}_{j}\right|^{2}\right)^{-1 / 2}\right\| \\
& \leq C \begin{cases}1+v_{j k}^{-\left(2-\gamma_{1} / 2\right)}\left|v_{j k} t\right|^{2-\gamma_{1}}, & \text { if } \zeta_{j k}^{a}, \\
1+v_{j k}^{-1} \ln \left(1+v_{j k}^{-1 / 2}\left|v_{j k} t\right|\right), & \text { if } \zeta_{j k}^{b}, \leq C\left(1+v_{j k}^{-\left(2-\gamma_{1} / 2\right)}\left|v_{j k} t\right|^{\theta_{j k}}\right), \\
1, & \text { if } \zeta_{j k}^{c},\end{cases} \\
B_{j k} & :=\left\|F\left(\left|\tilde{\mathbf{x}}_{k}-\tilde{\mathbf{x}}_{j}\right|>\kappa\left|v_{j k} t\right|\right) \tilde{U}_{D}(t) \prod_{j^{\prime}<k^{\prime}} f_{j^{\prime} k^{\prime}}\left(\mathbf{p}_{j^{\prime} k^{\prime}}-\mu_{j^{\prime} k^{\prime}} \mathbf{v}_{j^{\prime} k^{\prime}}\right)\left(1+\left|\tilde{\mathbf{x}}_{k}-\tilde{\mathbf{x}}_{j}\right|^{2}\right)^{-2}\right\| \\
& \leq C\left(1+\left|v_{j k} t\right|\right)^{-2-\tilde{\epsilon}} .
\end{aligned}
$$

Proof.

By (1.21) and (1.22), for $1 \leq a \leq 4$, multiplication by $\left(\tilde{\mathbf{x}}_{k}-\tilde{\mathbf{x}}_{j}\right)^{a}$ becomes derivatives in the $\mathbf{p}, \mathbf{p}_{k}, k=$ $3, \ldots, N$ variables.

The norm

$$
\left\|\left(\tilde{\mathbf{x}}_{k}-\tilde{\mathbf{x}}_{j}\right)^{a} \tilde{U}_{D}(t) \prod_{j^{\prime}<k^{\prime}} f_{j^{\prime} k^{\prime}}\left(\mathbf{p}_{j^{\prime} k^{\prime}}-\mu_{j^{\prime} k^{\prime}} \mathbf{v}_{j^{\prime} k^{\prime}}\right)\left(1+\left|\tilde{\mathbf{x}}_{k}-\tilde{\mathbf{x}}_{j}\right|^{2}\right)^{-a / 2}\right\|
$$

is bounded by a finite sum of terms of the form $C \prod_{b=1}^{a} I_{\beta_{b}}$, with $I_{\beta_{b}}=1$ if the multi-index $\beta_{b}=0$ and if $\left|\beta_{b}\right|>0$,

$$
I_{\beta_{b}}=\left\|\int_{0}^{t}|s|^{\left|\beta_{b}\right|}\left(D^{\beta_{b}} V_{j^{\prime} k^{\prime}}^{l}\right)\left(s\left(\mathbf{p}_{j^{\prime} k^{\prime}} / \mu_{j^{\prime} k^{\prime}}+\mathbf{v}_{j^{\prime} k^{\prime}}\right)+\mathbf{e}_{1} q_{j^{\prime} k^{\prime}} E s^{2} /\left(2 \mu_{j^{\prime} k^{\prime}}\right)\right) d s g_{j^{\prime} k^{\prime}}\left(\mathbf{p}_{j^{\prime} k^{\prime}}\right)\right\|,
$$

where $\left(j^{\prime}, k^{\prime}\right)$ is a pair of integers $1 \leq j^{\prime}<k^{\prime} \leq N$ such that $j^{\prime}=j$ or $j^{\prime}=k$ or $k^{\prime}=j$ or $k^{\prime}=k$ or $j^{\prime}+j=3$, $g_{j^{\prime} k^{\prime}} \in C_{0}^{\infty}\left(B_{\mu_{j^{\prime} k^{\prime}} \eta_{j^{\prime} k^{\prime}}}\right)$ and $g_{j^{\prime} k^{\prime}}=1$ in the support of $f_{j^{\prime} k^{\prime}}$. Note that $\sum_{b=1}^{a}\left|\beta_{b}\right| \leq a$.

Below, we take $\tilde{\sigma}=0$, if $q_{j^{\prime} k^{\prime}} \neq 0$ and $\tilde{\sigma}=1$, if $q_{j^{\prime} k^{\prime}}=0$. We define

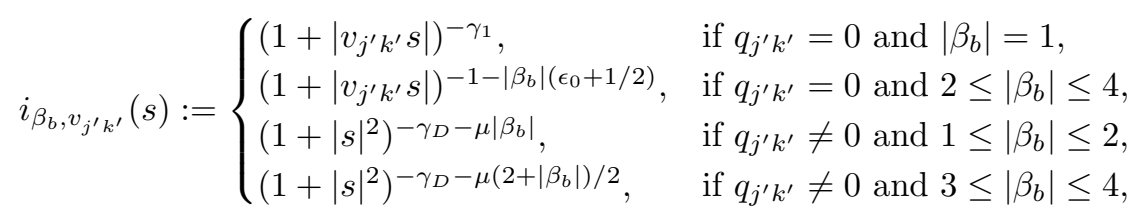

It follows from (1.24), (1.25), (1.29), (1.30), (2.13), (2.14), (2.18) and (2.19) that

$$
I_{\beta_{b}} \leq C \int_{0}^{|t|} s^{\left|\beta_{b}\right|} i_{\beta_{b}, v_{j^{\prime} k^{\prime}}}(s) d s \leq C v_{j^{\prime} k^{\prime}}^{-\left(\left|\beta_{b}\right|+1\right) \tilde{\sigma} /(2-\tilde{\sigma})} \int_{0}^{v_{j^{\prime} k^{\prime}}^{\tilde{\sigma} /(2-\tilde{\sigma})}|t|} \tau^{\left|\beta_{b}\right|} i_{\beta_{b}, 1}(\tau) d \tau .
$$

Let us assume $\left|\beta_{b}\right|=1$ in (2.20). If $q_{j^{\prime} k^{\prime}} \neq 0$,

$$
I_{\beta_{b}} \leq C \int_{0}^{|t|} \tau(1+\tau)^{2\left(-\gamma_{D}-\mu\right)} d \tau \leq C
$$

If $q_{j^{\prime} k^{\prime}}=0$, we have that

$$
I_{\beta_{b}} \leq C v_{j^{\prime} k^{\prime}}^{-2} \int_{0}^{v_{j^{\prime} k^{\prime}}|t|} \tau(1+\tau)^{-\gamma_{1}} d \tau \leq C v_{j^{\prime} k^{\prime}}^{-2} \begin{cases}\left(1+\left|v_{j^{\prime} k^{\prime}} t\right|\right)^{2-\gamma_{1}}, & \text { if } \gamma_{1}<2 \\ \ln \left(1+\left|v_{j^{\prime} k^{\prime}}\right|\right), & \text { if } \gamma_{1}=2\end{cases}
$$




$$
\leq C \begin{cases}1+v_{j k}^{-2}\left|v_{j k} t\right|^{2-\gamma_{1}}, & \text { if } \gamma_{1}<2 \text { and either }\left(j^{\prime}, k^{\prime}\right)=(j, k)=(1,2), \\ 1+v_{j k}^{-\left(2+\gamma_{1}\right)}\left|v_{j k} t\right|^{2-\gamma_{1}}, & \text { or }\left(j^{\prime}, k^{\prime}\right) \neq(1,2) \text { and }(j, k) \neq(1,2), \\ 1+v_{j k}^{-\left(2-\gamma_{1} / 2\right)}\left|v_{j k} t\right|^{2-\gamma_{1}}, & \text { if } \gamma_{1}<2,\left(j^{\prime}, k^{\prime}\right) \neq(1,2) \text { and }(j, k)=(1,2), \\ v_{j k}^{-2} \ln \left(1+\left|v_{j k} t\right|\right), & \text { if } \gamma_{1}=2 \text { and either }\left(j^{\prime}, k^{\prime}\right)=(j, k)=(1,2), \\ & \text { or }\left(j^{\prime}, k^{\prime}\right) \neq(1,2) \text { and }(j, k) \neq(1,2), \\ v_{j k}^{-4} \ln \left(1+\left|v_{j k}^{2} t\right|\right), & \text { if } \gamma_{1}=2,\left(j^{\prime}, k^{\prime}\right) \neq(1,2) \text { and }(j, k)=(1,2), \\ v_{j k}^{-1} \ln \left(1+\left|v_{j k}^{1 / 2} t\right|\right), & \text { if } \gamma_{1}=2,\left(j^{\prime}, k^{\prime}\right)=(1,2) \text { and }(j, k) \neq(1,2) .\end{cases}
$$

This implies that (2.16) is true.

In the other hand, similarly to (2.20), we have that,

$$
\begin{aligned}
I_{\beta_{b}} & \leq C \int_{0}^{|t|} s^{\left|\beta_{b}\right|} i_{\beta_{b}, v}(s) d s \leq C v^{-\left(\left|\beta_{b}\right|+1\right) \tilde{\sigma} /(2-\tilde{\sigma})} \int_{0}^{v^{\tilde{\sigma} /(2-\tilde{\sigma})}|t|} \tau^{\left|\beta_{b}\right|} i_{\beta_{b}, 1}(\tau) d \tau \\
& \leq C \begin{cases}1+|v t|^{\left|\beta_{b}\right|\left(-\epsilon_{0}+1 / 2\right)}, & \text { if } q_{j^{\prime} k^{\prime}}=0 \text { and } 1 \leq\left|\beta_{b}\right| \leq 4, \\
1, & \text { if } q_{j^{\prime} k^{\prime}} \neq 0 \text { and } 1 \leq\left|\beta_{b}\right| \leq 2, \\
1+|v t|^{\max \left\{\left|\beta_{b}\right|+1-2 \gamma_{D}-\left(\left|\beta_{b}\right|+2\right) \mu, 0\right\}}, & \text { if } q_{j^{\prime} k^{\prime}} \neq 0 \text { and } 3 \leq\left|\beta_{b}\right| \leq 4 .\end{cases}
\end{aligned}
$$

Then, it follows that

$$
C \prod_{b=1}^{4} I_{\beta_{b}} \leq C(1+|v t|)^{2-\tilde{\epsilon}}
$$

hence

$$
\begin{aligned}
\kappa^{4}\left|v_{j k} t\right|^{4} B_{j k} & \leq\left\|\left|\tilde{\mathbf{x}}_{k}-\tilde{\mathbf{x}}_{j}\right|^{4} F\left(\left|\tilde{\mathbf{x}}_{k}-\tilde{\mathbf{x}}_{j}\right|>\kappa\left|v_{j k} t\right|\right) \tilde{U}_{D}(t) \prod_{j^{\prime}<k^{\prime}} f_{j^{\prime} k^{\prime}}\left(\mathbf{p}_{j^{\prime} k^{\prime}}-\mu_{j^{\prime} k^{\prime}} \mathbf{v}_{j^{\prime} k^{\prime}}\right)\left(1+\left|\tilde{\mathbf{x}}_{k}-\tilde{\mathbf{x}}_{j}\right|^{2}\right)^{-2}\right\| \\
& \leq C(1+|v t|)^{2-\tilde{\epsilon}} \leq C\left(1+\left|v_{j k} t\right|\right)^{2-\tilde{\epsilon}} .
\end{aligned}
$$

This proves Equation (2.17).

Lemma (2.3), below, is a generalization of equations (3.8) and (3.17) in Weder [39]. Note that conditions (1.23) and (1.26) imply that

$$
\left\|V^{v s}(\mathbf{x}) g(\mathbf{p}) F(|\mathbf{x}| \geq R)\right\|
$$

is an integrable function of $\mathrm{R}$ for all $g \in C_{0}^{\infty}\left(\mathbb{R}^{n}\right)$ (see Corollary 2.4 in Enss [14). It follows that potentials in $\mathcal{V}_{E, v s}$ and $\mathcal{V}_{0, v s}$, satisfy condition (2.21) below with $\rho=0$. Of course, larger $\rho$ means faster decay.

LEMMA 2.3. Suppose that $V_{j k}^{v s}$ is given as in (2.1) and satisfies

$$
(1+R)^{\rho}\left\|V_{j k}^{v s}\left(\tilde{\mathbf{x}}_{k}-\tilde{\mathbf{x}}_{j}\right) g\left(\mathbf{p}_{j k}\right) F\left(\left|\tilde{\mathbf{x}}_{k}-\tilde{\mathbf{x}}_{j}\right| \geq R\right)\right\| \in L^{1}((0, \infty), d R),
$$

for some $0 \leq \rho \leq 1$ and all $g \in C_{0}^{\infty}\left(\mathbb{R}^{n}\right), \tilde{U}_{D}(t)$ is given as in (2.3). Then, for all functions $f_{j^{\prime} k^{\prime}} \in$ $C_{0}^{\infty}\left(B_{\mu_{j^{\prime} k^{\prime}} \eta_{j^{\prime} k^{\prime}}}\right)$ with $1 \leq j^{\prime}<k^{\prime} \leq N$, there is a function $h_{j k}$ with $(1+\tau)^{\rho} h_{j k}(\tau) \in L^{1}((0, \infty))$ such that for every $\mathbf{v}_{j k} \in \mathbb{R}^{n}$ with $v_{j k}>c$ for some constant $0<c$, we have the following estimate, for all integers $1 \leq j<k \leq N$ :

$$
D_{j k}:=\left\|V_{j k}^{v s}\left(\tilde{\mathbf{x}}_{k}-\tilde{\mathbf{x}}_{j}\right) e^{-i t H_{0}} \tilde{U}_{D}(t) \prod_{j^{\prime}<k^{\prime}} f_{j^{\prime} k^{\prime}}\left(\mathbf{p}_{j^{\prime} k^{\prime}}-\mu_{j^{\prime} k^{\prime}} \mathbf{v}_{j^{\prime} k^{\prime}}\right)\left(1+\left|\tilde{\mathbf{x}}_{k}-\tilde{\mathbf{x}}_{j}\right|^{2}\right)^{-2}\right\| \leq h_{j k}\left(\left|v_{j k} t\right|\right) .
$$


Proof. Let us take $g_{j k} \in C_{0}^{\infty}\left(B_{\mu_{j k} \eta_{j k}}\right)$ that satisfies $g_{j k} \equiv 1$ on the support of $f_{j k}$.

$$
D_{j k} \leq I_{1}+I_{2}+I_{3}
$$

where, for any positive constant $\lambda$,

$$
\begin{aligned}
& I_{1}=\left\|V_{j k}^{v s}\left(\tilde{\mathbf{x}}_{k}-\tilde{\mathbf{x}}_{j}\right) g_{j k}\left(\mathbf{p}_{j k}-\mu_{j k} \mathbf{v}_{j k}\right)\right\| \| F\left(\left|\tilde{\mathbf{x}}_{k}-\tilde{\mathbf{x}}_{j}-\mathbf{v}_{j k} t-\mathbf{e}_{1} q_{j k} E t^{2} /\left(2 \mu_{j k}\right)\right| \geq \lambda\left|v_{j k} t\right| 5 / 8\right) e^{-i t H_{0}} \\
& \times g_{j k}\left(\mathbf{p}_{j k}-\mu_{j k} \mathbf{v}_{j k}\right) F\left(\left|\tilde{\mathbf{x}}_{k}-\tilde{\mathbf{x}}_{j}\right|<\lambda\left|v_{j k} t\right| / 8\right) \| \\
& \times\left\|\tilde{U}_{D}(t) \prod_{j^{\prime}<k^{\prime}} f_{j^{\prime} k^{\prime}}\left(\mathbf{p}_{j^{\prime} k^{\prime}}-\mu_{j^{\prime} k^{\prime}} \mathbf{v}_{j^{\prime} k^{\prime}}\right)\left(1+\left|\tilde{\mathbf{x}}_{k}-\tilde{\mathbf{x}}_{j}\right|^{2}\right)^{-2}\right\|, \\
& I_{2}=\left\|V_{j k}^{v s}\left(\tilde{\mathbf{x}}_{k}-\tilde{\mathbf{x}}_{j}\right) g_{j k}\left(\mathbf{p}_{j k}-\mu_{j k} \mathbf{v}_{j k}\right) F\left(\left|\tilde{\mathbf{x}}_{k}-\tilde{\mathbf{x}}_{j}-\mathbf{v}_{j k} t-\mathbf{e}_{1} q_{j k} E t^{2} /\left(2 \mu_{j k}\right)\right| \geq \lambda\left|v_{j k} t\right| 5 / 8\right) e^{-i t H_{0}}\right\| \\
& \times\left\|F\left(\left|\tilde{\mathbf{x}}_{k}-\tilde{\mathbf{x}}_{j}\right| \geq \lambda\left|v_{j k} t\right| / 8\right) \tilde{U}_{D}(t) \prod_{j^{\prime}<k^{\prime}} f_{j^{\prime} k^{\prime}}\left(\mathbf{p}_{j^{\prime} k^{\prime}}-\mu_{j^{\prime} k^{\prime}} \mathbf{v}_{j^{\prime} k^{\prime}}\right)\left(1+\left|\tilde{\mathbf{x}}_{k}-\tilde{\mathbf{x}}_{j}\right|^{2}\right)^{-2}\right\|, \\
& I_{3}=\left\|V_{j k}^{v s}\left(\tilde{\mathbf{x}}_{k}-\tilde{\mathbf{x}}_{j}\right) g_{j k}\left(\mathbf{p}_{j k}-\mu_{j k} \mathbf{v}_{j k}\right) F\left(\left|\tilde{\mathbf{x}}_{k}-\tilde{\mathbf{x}}_{j}-\mathbf{v}_{j k} t-\mathbf{e}_{1} q_{j k} E t^{2} /\left(2 \mu_{j k}\right)\right|<\lambda\left|v_{j k} t\right| 5 / 8\right)\right\| \\
& \times\left\|e^{-i t H_{0}} \tilde{U}_{D}(t) \prod_{j^{\prime}<k^{\prime}} f_{j^{\prime} k^{\prime}}\left(\mathbf{p}_{j^{\prime} k^{\prime}}-\mu_{j^{\prime} k^{\prime}} \mathbf{v}_{j^{\prime} k^{\prime}}\right)\left(1+\left|\tilde{\mathbf{x}}_{k}-\tilde{\mathbf{x}}_{j}\right|^{2}\right)^{-2}\right\| .
\end{aligned}
$$

We give the proof for the pair $(1,2)$, the other cases are similar, using Jacobi coordinates based in the pair $(j, k)$. Let us set $\mathbf{x}=\tilde{\mathbf{x}}_{2}-\tilde{\mathbf{x}}_{1}, \mathbf{p}=-i \nabla_{\mathbf{x}}$ we obtain as in (1.7) that

$$
H_{0}=\left[\left(2 \nu_{1}\right)^{-1} \mathbf{p}^{2}+q_{12} \mathbf{E} \cdot \mathbf{x}\right] \otimes I+I \otimes \hat{H}_{0},
$$

where $I \otimes \hat{H}_{0}$ conmutes with $\mathbf{x}$, by virtue of $\hat{H}_{0}$ 's independence from $\mathbf{x}$. Note that $\nu_{1}=\mu_{12}$. Let us write $v=v_{12}=|\mathbf{v}|$. Therefore, thanks to commutativity

$$
e^{-i t H_{0}}=e^{-i t\left[\left(2 \nu_{1}\right)^{-1} \mathbf{p}^{2}+q_{12} \mathbf{E} \cdot \mathbf{x}\right] \otimes I-i t I \otimes \hat{H}_{0}}=e^{-i t\left[\left(2 \nu_{1}\right)^{-1} \mathbf{p}^{2}+q_{12} \mathbf{E} \cdot \mathbf{x}\right]} \otimes e^{-i t \hat{H}_{0}} .
$$

We observe that the second factor in the tensorial product above conmutes with any operator depending on $\mathbf{x}$ and $\mathbf{p}$. It is also unitary, thus it disappears from the following norm estimations. We define $\mathcal{M}^{\prime}=\{\mathbf{x} \in$ $\left.\mathbb{R}^{n}|| \mathbf{x}-\mathbf{v} t|\geq \lambda| v t \mid 5 / 8\right\}$ and $\mathcal{M}=\left\{\mathbf{x} \in \mathbb{R}^{n}|| \mathbf{x}|<\lambda| v t \mid / 8\right\}$. We proceed as in Weder [39] using (1.31)-(1.34).

$$
\begin{aligned}
I_{1} \leq & C\left\|F\left(\left|\mathbf{x}-\mathbf{v} t-\mathbf{e}_{1} q_{12} E t^{2} /\left(2 \mu_{12}\right)\right| \geq \lambda|v t| 5 / 8\right) e^{-i t H_{0}} g_{12}\left(\mathbf{p}-\mu_{12} \mathbf{v}\right) F(|\mathbf{x}|<\lambda|v t| / 8)\right\| \\
= & C \| F\left(\left|\mathbf{x}-\mathbf{v} t-\mathbf{e}_{1} q_{12} E t^{2} /\left(2 \mu_{12}\right)\right| \geq \lambda|v t| 5 / 8\right) e^{-i \mathbf{p} \cdot \mathbf{e}_{1} q_{12} E t^{2} /\left(2 \mu_{12}\right)} \\
& \quad \times e^{-i t \mathbf{p}^{2} /\left(2 \mu_{12}\right)} g_{12}\left(\mathbf{p}-\mu_{12} \mathbf{v}\right) F(|\mathbf{x}|<\lambda|v t| / 8) \| \\
= & C\left\|F(|\mathbf{x}-\mathbf{v} t| \geq \lambda|v t| 5 / 8) e^{-i t \mathbf{p}^{2} /\left(2 \mu_{12}\right)} g_{12}\left(\mathbf{p}-\mu_{12} \mathbf{v}\right) F(|\mathbf{x}|<\lambda|v t| / 8)\right\| \\
= & C\left\|F\left(\mathbf{x} \in \mathcal{M}^{\prime}\right) e^{-i t \mathbf{p}^{2} /\left(2 \mu_{12}\right)} g_{12}\left(\mathbf{p}-\mu_{12} \mathbf{v}\right) F(\mathbf{x} \in \mathcal{M})\right\| \\
\leq & C(1+\lambda|v t| / 4+|t|)^{-3} \leq C(1+|v t|)^{-3} .
\end{aligned}
$$

To justify (2.24), we will prove that $r \geq \lambda|v t| / 4$ in Proposition 2.1, provided $v>4 \eta / \lambda$. Let us take $x \in \mathcal{M}^{\prime}$ and $y \in \mathcal{M}+\mathbf{v} t$, then $|\mathbf{x}-\mathbf{y}|=|(\mathbf{x}-\mathbf{v} t)-(\mathbf{y}-\mathbf{v} t)| \geq \lambda|v t| 5 / 8-\lambda|v t| / 8=\lambda|v t| / 2$. Thus, $r \geq \lambda|v t| / 2-\eta|t| \geq \lambda|v t| / 2-\lambda|v t| / 4$. 
Application of Lemma 2.2, equation (2.17), yields for an $\epsilon>0$,

$$
I_{2} \leq C(1+|v t|)^{-2-\epsilon} \text {. }
$$

Then, by (2.13), $\quad I_{3} \leq C\left\|V_{12}^{v s}(\mathbf{x}) g(\mathbf{p}) F\left(\left|\mathbf{x}-\mathbf{v} t-\mathbf{e}_{1} q_{12} E t^{2} /\left(2 \mu_{12}\right)\right|<\lambda|v t| 5 / 8\right)\right\|$

$\leq C\left\|V_{12}^{v s}(\mathbf{x}) g(\mathbf{p}) F\left(|\mathbf{x}| \geq|v t|\left(\sqrt{\delta_{1}}-5 \lambda / 8\right)\right)\right\|$

$:=h_{12}(|v t|)$,

where, by (2.21), $h_{12}(\tau) \in L^{1}((0, \infty))$, provided $\lambda<8 \sqrt{\delta_{1}} / 5$.

Inequalities (2.23), (2.24), (2.25) and (2.26) prove the Lemma.

LEMMA 2.4. Given $V_{j k}^{E, s} \in \mathcal{V}_{E, s}$, where $1 \leq j<k \leq N, \alpha$ as in Definition [1.2, $\tilde{U}_{D}(t)$ be given as in (2.3). Then for all functions $f_{j^{\prime} k^{\prime}} \in C_{0}^{\infty}\left(B_{\mu_{j^{\prime} k^{\prime}} \eta_{j^{\prime} k^{\prime}}}\right)$ with $1 \leq j^{\prime}<k^{\prime} \leq N$, there is a constant $0<c$ such that for every $\mathbf{v}_{j k} \in \mathbb{R}^{n}$ with $v_{j k}>c$, the following estimate is true for all $0<\epsilon_{1}<1$ :

$$
\begin{aligned}
& \int_{-\infty}^{\infty} d t \|\left(V_{j k}^{E, s}\left(\tilde{\mathbf{x}}_{k}-\tilde{\mathbf{x}}_{j}\right)-V_{j k}^{E, s}\left(\mathbf{v}_{j k} t+\mathbf{e}_{1} q_{j k} E t^{2} /\left(2 \mu_{j k}\right)\right)\right) \\
& \times e^{-i t H_{0}} \tilde{U}_{D}(t) \prod_{j^{\prime}<k^{\prime}} f_{j^{\prime} k^{\prime}}\left(\mathbf{p}_{j^{\prime} k^{\prime}}-\mu_{j^{\prime} k^{\prime}} \mathbf{v}_{j^{\prime} k^{\prime}}\right)\left(1+\left|\tilde{\mathbf{x}}_{k}-\tilde{\mathbf{x}}_{j}\right|^{2}\right)^{-2} \|= \begin{cases}O\left(v_{j k}^{-\alpha}\right), & \text { if } \alpha<1, \\
O\left(v_{j k}^{-1+\epsilon_{1}}\right), & \text { if } \alpha=1 .\end{cases}
\end{aligned}
$$

Proof. The proof is quite similar to that of Lemma 2.2 in Adachi and Maehara 2]. To simplify the notation let us assume, in this Lemma, that $q_{12} \neq 0$ and consider the pair $(1,2)$, i.e. $\mathbf{x}=\tilde{\mathbf{x}}_{2}-\tilde{\mathbf{x}}_{1}, \mathbf{p}=-i \nabla_{\mathbf{x}}$. Let us take $g_{12} \in C_{0}^{\infty}\left(B_{\mu_{12} \eta}\right)$ that satisfies $g_{12} \equiv 1$ on the support of $f_{12}$.

We simplify as follows, noting that $V_{12}^{E, s}$ is bounded:

$$
\begin{aligned}
I & =\left\|\left(V_{12}^{E, s}(\mathbf{x})-V_{12}^{E, s}\left(\mathbf{v} t+\mathbf{e}_{1} q_{12} E t^{2} /\left(2 \mu_{12}\right)\right)\right) e^{-i t H_{0}} \tilde{U}_{D}(t) \prod_{j^{\prime}<k^{\prime}} f_{j^{\prime} k^{\prime}}\left(\mathbf{p}_{j^{\prime} k^{\prime}}-\mu_{j^{\prime} k^{\prime}} \mathbf{v}_{j^{\prime} k^{\prime}}\right)\left(1+\mathbf{x}^{2}\right)^{-2}\right\| \\
& \leq C\left(I_{1}+I_{2}+I_{3}\right),
\end{aligned}
$$

where, for $0<\tilde{\alpha}<1$,

$$
\begin{aligned}
I_{1} & =\left\|F\left(\left|\mathbf{x}-\mathbf{v} t-\mathbf{e}_{1} q_{12} E t^{2} /\left(2 \mu_{12}\right)\right| \geq 3\left|v^{\tilde{\alpha}} t\right|\right) e^{-i t H_{0}} g_{12}\left(\mathbf{p}-\mu_{12} \mathbf{v}\right) F\left(|\mathbf{x}|<\left|v^{\tilde{\alpha}} t\right|\right)\right\| \\
& =\left\|F\left(|\mathbf{x}-\mathbf{v} t| \geq 3\left|v^{\tilde{\alpha}} t\right|\right) e^{-i t \mathbf{p}^{2} /\left(2 \mu_{12}\right)} g_{12}\left(\mathbf{p}-\mu_{12} \mathbf{v}\right) F\left(|\mathbf{x}|<\left|v^{\tilde{\alpha}} t\right|\right)\right\|, \\
I_{2} & =\left\|F\left(|\mathbf{x}| \geq\left|v^{\tilde{\alpha}} t\right|\right) \tilde{U}_{D}(t) \prod_{j^{\prime}<k^{\prime}} f_{j^{\prime} k^{\prime}}\left(\mathbf{p}_{j^{\prime} k^{\prime}}-\mu_{j^{\prime} k^{\prime}} \mathbf{v}_{j^{\prime} k^{\prime}}\right)\left(1+\mathbf{x}^{2}\right)^{-2}\right\|, \\
I_{3} & =\left\|\left(V_{12}^{E, s}(\mathbf{x})-V_{12}^{E, s}\left(\mathbf{v} t+\mathbf{e}_{1} q_{12} E t^{2} /\left(2 \mu_{12}\right)\right)\right) F\left(\left|\mathbf{x}-\mathbf{v} t-\mathbf{e}_{1} q_{12} E t^{2} /\left(2 \mu_{12}\right)\right|<3\left|v^{\tilde{\alpha}} t\right|\right)\right\| \\
& =\left\|\left(V_{12}^{E, s}\left(\mathbf{x}+\mathbf{v} t+\mathbf{e}_{1} q_{12} E t^{2} /\left(2 \mu_{12}\right)\right)-V_{12}^{E, s}\left(\mathbf{v} t+\mathbf{e}_{1} q_{12} E t^{2} /\left(2 \mu_{12}\right)\right)\right) F\left(|\mathbf{x}|<3\left|v^{\tilde{\alpha}} t\right|\right)\right\| .
\end{aligned}
$$

$I_{1}$ and $I_{2}$ are estimated as in the proof of Lemma 2.3, by Proposition 2.1 and equation 2.17, respectively:

$$
\int_{-\infty}^{\infty}\left(I_{1}+I_{2}\right) d t=O\left(v^{-\tilde{\alpha}}\right)
$$

By lemma 2.2 of Adachi and Maehara 2] (see also page 042101-5, equation 2.10 of [2]), we get, for all $0<\tilde{\alpha}<1$ and $v$ sufficiently large that

$$
\int_{-\infty}^{\infty} I_{3} d t= \begin{cases}O\left(v^{\tilde{\alpha}-2 \alpha}\right), & \text { if } \alpha<1 \\ O\left(v^{\tilde{\alpha}-2}|\ln v|\right), & \text { if } \alpha=1\end{cases}
$$


We finish the proof by setting $\tilde{\alpha}= \begin{cases}\alpha, & \text { if } \alpha<1, \\ 1-\epsilon_{1}, & \text { if } \alpha=1,0<\epsilon_{1}<1 .\end{cases}$

LEMMA 2.5. Let $V_{j k}^{l}$ and $\tilde{U}_{D}(t)$ be given as in (2.1) and (2.3), respectively. Let $\gamma_{1}, \epsilon_{0}$ be as in Definition 1.1. $\gamma_{D}, \mu$ be as in Definition 1.2, $\theta_{j k}$ as in (2.15). Let us define two constants $\sigma_{j k}$ and $\tilde{\sigma}_{j k}$; if $q_{j k} \neq 0$ and $V_{j k}^{l} \neq 0$, then $\sigma_{j k}=\frac{\tilde{\sigma}_{j k}}{2-\tilde{\sigma}_{j k}}$ and $0<\tilde{\sigma}_{j k}<2-\max \left\{\frac{1+\theta_{j k}}{\gamma_{D}+\mu}, \frac{2}{\gamma_{D}+2 \mu}, 1\right\}$, else, if $q_{j k}=0$ or $V_{j k}^{l}=0$, then $\sigma_{j k}:=\tilde{\sigma}_{j k}:=1$. Then for all functions $f_{j^{\prime} k^{\prime}} \in C_{0}^{\infty}\left(B_{\mu_{j^{\prime} k^{\prime}} \eta_{j^{\prime} k^{\prime}}}\right)$ with $1 \leq j^{\prime}<k^{\prime} \leq N$, and for all integers $1 \leq j<k \leq N$, there is a constant $v_{0}>0$ such that for every $\mathbf{v}_{j k} \in \mathbb{R}^{n}$ with $v_{j k}>v_{0}^{1 / \sigma_{j k}}$, we have the following estimate:

$$
\begin{gathered}
\int_{-\infty}^{\infty} d t \|\left(V_{j k}^{l}\left(\tilde{\mathbf{x}}_{k}-\tilde{\mathbf{x}}_{j}\right)-V_{j k}^{l}\left(t \mathbf{p}_{j k} / \mu_{j k}-\mathbf{e}_{1} q_{j k} E t^{2} /\left(2 \mu_{j k}\right)\right)\right) \\
\times e^{-i t H_{0}} \tilde{U}_{D}(t) \prod_{j^{\prime}<k^{\prime}} f_{j^{\prime} k^{\prime}}\left(\mathbf{p}_{j^{\prime} k^{\prime}}-\mu_{j^{\prime} k^{\prime}} \mathbf{v}_{j^{\prime} k^{\prime}}\right)\left(1+\left|\tilde{\mathbf{x}}_{k}-\tilde{\mathbf{x}}_{j}\right|^{2}\right)^{-2} \| \leq O\left(v_{j k}^{-\sigma_{j k}}\right) .
\end{gathered}
$$

Proof. The proof in the case where $q_{j k}=0$ is quite similar to that of Lemma 3.3 in Enss and Weder [19, and the proof in the case where $q_{j k} \neq 0$ is quite similar to that of Lemma 3.4 in Adachi and Maehara [2]. In this Lemma, let us denote $\mathbf{x}=\tilde{\mathbf{x}}_{k}-\tilde{\mathbf{x}}_{j}, \mathbf{p}=-i \nabla_{\mathbf{x}}$. From (2.14), a constant is defined as follows

$$
c:= \begin{cases}c_{1}^{\tilde{\sigma}_{j k}} c_{2}^{1-\tilde{\sigma}_{j k},} & \text { if } q_{j k} \neq 0, \\ 1 / 2, & \text { if } q_{j k}=0 .\end{cases}
$$

Let's split the long-range potential $V_{j k}^{l}$ into two parts with controllable decay properties. Let $\chi \in$ $C^{\infty}\left(\mathbb{R}^{n}\right)$ satisfy, $0 \leq \chi \leq 1, \chi(\mathbf{u})=1$, for $|\mathbf{u}| \geq c$ and $\chi(\mathbf{u})=0$, for $|\mathbf{u}| \leq c / 2$; and $V_{j k, v_{j k} t}^{l}(\mathbf{u})=$ $V_{j k}^{l}(\mathbf{u}) \chi\left(\mathbf{u} /\left(v_{j k}^{\tilde{\sigma}_{j k}}|t|^{2-\tilde{\sigma}_{j k}}\right)\right)$. In consequence, $\operatorname{supp}\left(V_{j k, v_{j k} t}^{l}-V_{j k}^{l}\right) \subset B_{c v_{j k}}^{\tilde{\sigma}_{j k}|t|^{2-\tilde{\sigma}_{j k}}}$, and $\left\|V_{j k, v_{j k} t}^{l}-V_{j k}^{l}\right\| \leq$ $\left\|V_{j k}^{l}\right\|$.

Choosing again $g \in C_{0}^{\infty}\left(B_{\mu_{j k}} \eta_{j k}\right)$ such that $g \equiv 1$ on the support of $f_{j k}$, it follows that

$$
\begin{aligned}
& \left\|\left(V_{j k}^{l}(\mathbf{x})-V_{j k}^{l}\left(t \mathbf{p} / \mu_{j k}-\mathbf{e}_{1} q_{j k} E t^{2} /\left(2 \mu_{j k}\right)\right)\right) e^{-i t H_{0}} \tilde{U}_{D}(t) \prod_{j^{\prime}<k^{\prime}} f_{j^{\prime} k^{\prime}}\left(\mathbf{p}_{j^{\prime} k^{\prime}}-\mu_{j^{\prime} k^{\prime}} \mathbf{v}_{j^{\prime} k^{\prime}}\right)\left(1+\mathbf{x}^{2}\right)^{-2}\right\| \\
\leq & I_{1}+I_{2}+I_{3},
\end{aligned}
$$

where

$$
\begin{aligned}
& I_{1}=\|\left(V_{j k, v_{j k} t}^{l}(\mathbf{x})-V_{j k}^{l}\left(t \mathbf{p} / \mu_{j k}-\mathbf{e}_{1} q_{j k} E t^{2} /\left(2 \mu_{j k}\right)\right)\right) e^{-i t H_{0}} g\left(\mathbf{p}-\mu_{j k} \mathbf{v}_{j k}\right) \\
& \times \tilde{U}_{D}(t) \prod_{j^{\prime}<k^{\prime}} f_{j^{\prime} k^{\prime}}\left(\mathbf{p}_{j^{\prime} k^{\prime}}-\mu_{j^{\prime} k^{\prime}} \mathbf{v}_{j^{\prime} k^{\prime}}\right)\left(1+\mathbf{x}^{2}\right)^{-2} \|, \\
& I_{2}=\left\|\left(V_{j k}^{l}-V_{j k, v_{j k} t}^{l}\right)(\mathbf{x}) e^{-i t H_{0}} g\left(\mathbf{p}-\mu_{j k} \mathbf{v}_{j k}\right) F\left(|\mathbf{x}|<v_{j k}^{\sigma_{j k}}|t| / 8\right)\right\| \\
& \times\left\|\tilde{U}_{D}(t) \prod_{j^{\prime}<k^{\prime}} f_{j^{\prime} k^{\prime}}\left(\mathbf{p}_{j^{\prime} k^{\prime}}-\mu_{j^{\prime} k^{\prime}} \mathbf{v}_{j^{\prime} k^{\prime}}\right)\left(1+\mathbf{x}^{2}\right)^{-2}\right\|,
\end{aligned}
$$




$$
\begin{aligned}
I_{3}= & \left\|\left(V_{j k}^{l}-V_{j k, v_{j k}}^{l}\right)(\mathbf{x}) e^{-i t H_{0}} g\left(\mathbf{p}-\mu_{j k} \mathbf{v}_{j k}\right)\right\| \\
& \times\left\|F\left(|\mathbf{x}| \geq v_{j k}^{\sigma_{j k}}|t| / 8\right) \tilde{U}_{D}(t) \prod_{j^{\prime}<k^{\prime}} f_{j^{\prime} k^{\prime}}\left(\mathbf{p}_{j^{\prime} k^{\prime}}-\mu_{j^{\prime} k^{\prime}} \mathbf{v}_{j^{\prime} k^{\prime}}\right)\left(1+\mathbf{x}^{2}\right)^{-2}\right\| .
\end{aligned}
$$

If $q_{j k} \neq 0$, for $\mathbf{q} \in B_{\mu_{j k} \eta_{j k}}, v_{0} \geq 4 \eta_{j k} / \sqrt{1-\delta_{j k}}$, by (2.14), we have

$$
\left|t \mathbf{q} / \mu_{j k}+\mathbf{v}_{j k} t+\mathbf{e}_{1} q_{j k} E t^{2} /\left(2 \mu_{j k}\right)\right| \geq c v_{j k}^{\tilde{\sigma}_{j k}}|t|^{2-\tilde{\sigma}_{j k}} .
$$

If $q_{j k} \neq 0$ with $\mathbf{p}$ in the support of $g$, we note, by (2.33), that $V_{j k, v_{j k} t}^{l}\left(t \mathbf{p} / \mu_{j k}+\mathbf{v}_{j k} t+\mathbf{e}_{1} q_{j k} E t^{2} /\left(2 \mu_{j k}\right)\right)=$ $V_{j k}^{l}\left(t \mathbf{p} / \mu_{j k}+\mathbf{v}_{j k} t+\mathbf{e}_{1} q_{j k} E t^{2} /\left(2 \mu_{j k}\right)\right)$. If $q_{j k}=0$, $\mathbf{p}$ belongs to the support of $g\left(\cdot-\mu_{j k} \mathbf{v}_{j k}\right)$, and $v_{0}>2 \eta_{j k}$ then $V_{j k, v_{j k} t}^{l}\left(t \mathbf{p} / \mu_{j k}\right)=V_{j k}^{l}\left(t \mathbf{p} / \mu_{j k}\right)$.

As in Enss and Weder [19] and Adachi and Maehara 2], by (1.31)-(1.35) and the Baker-CampbellHausdorff formula [13],

$$
\begin{aligned}
I_{1} \leq \int_{0}^{1} d s & \|\left[\left(\nabla V_{j k, v_{j k} t}^{l}\right)\left(s \mathbf{x}+t \mathbf{p} / \mu_{j k}+\mathbf{v}_{j k} t+\mathbf{e}_{1} q_{j k} E t^{2} /\left(2 \mu_{j k}\right)\right) \cdot \mathbf{x}\right. \\
& \left.+\frac{i t}{\left(2 \mu_{j k}\right)}\left(\Delta V_{j k, v_{j k} t}^{l}\right)\left(s \mathbf{x}+t \mathbf{p} / \mu_{j k}+\mathbf{v}_{j k} t+\mathbf{e}_{1} q_{j k} E t^{2} /\left(2 \mu_{j k}\right)\right)\right] g(\mathbf{p}) e^{-i \mu_{j k} \mathbf{v}_{j k} \cdot \mathbf{x}} \\
& \times \tilde{U}_{D}(t) \prod_{j^{\prime}<k^{\prime}} f_{j^{\prime} k^{\prime}}\left(\mathbf{p}_{j^{\prime} k^{\prime}}-\mu_{j^{\prime} k^{\prime}} \mathbf{v}_{j^{\prime} k^{\prime}}\right)\left(1+\mathbf{x}^{2}\right)^{-2} \|
\end{aligned}
$$

For $q_{j k}=0, \epsilon_{0}<\gamma_{1}-3 / 2$, having in consideration that in the support of $V_{j k, v_{j k} t}^{l}$ we must have $|\mathbf{x}| \geq(c / 2)\left|v_{j k} t\right|,(2.16)$ and (2.34) imply that

$$
I_{1} \leq C\left[\left|v_{j k} t\right|^{-\gamma_{1}}\left(1+v_{j k}^{-\left(2-\gamma_{1} / 2\right)}\left|v_{j k} t\right|^{\theta_{j k}}\right)+\left|v_{j k} t\right|^{-1-2 \epsilon_{0}}\right] \leq C\left|v_{j k} t\right|^{-1-2 \epsilon_{0}} .
$$

Then, by the fact that $I_{1}$ is uniformly bounded, for all $t \in \mathbb{R}$ and all $v_{j k}, \int d t I_{1}=O\left(v_{j k}^{-1}\right)$.

We consider now the case when $q_{j k} \neq 0$. Recall that in the support of $V_{j k, v_{j k} t}^{l}$ we must have $|\mathbf{x}| \geq$ $(c / 2) v_{j k}^{\tilde{\sigma}_{j k}}|t|^{2-\tilde{\sigma}_{j k}}$ for $0<\tilde{\sigma}_{j k}<1$. For $0<b$, (2.16) and (2.34) imply:

$$
\begin{gathered}
I_{1} \leq I_{11}+I_{12}, \text { where, } I_{12} \leq C\left\|V_{j k}^{l}\right\|, \\
I_{11} \leq \quad C\left(\left(v_{j k}^{-\tilde{\sigma}_{j k}\left(\gamma_{D}+\mu\right)}|t|^{-\left(2-\tilde{\sigma}_{j k}\right)\left(\gamma_{D}+\mu\right)}+v_{j k}^{-\tilde{\sigma}_{j k}\left(\gamma_{D}+1\right)}|t|^{-\left(2-\tilde{\sigma}_{j k}\right)\left(\gamma_{D}+1\right)}\right)\right. \\
\left.\times\left(1+v_{j k}^{-\left(2-\gamma_{1} / 2\right)}\left|v_{j k} t\right|^{\theta_{j k}}\right) F\left(|t|>v_{j k}^{-b}\right)+\left\|V_{j k}^{l}\right\| F\left(|t| \leq v_{j k}^{-b}\right)\right) \text { and } \\
I_{12} \leq C\left(v_{j k}^{-\tilde{\sigma}_{j k}\left(\gamma_{D}+2 \mu\right)}|t|^{-\left(2-\tilde{\sigma}_{j k}\right)\left(\gamma_{D}+2 \mu\right)+1}+v_{j k}^{-\tilde{\sigma}_{j k}\left(\gamma_{D}+\mu+1\right)}|t|^{-\left(2-\tilde{\sigma}_{j k}\right)\left(\gamma_{D}+\mu+1\right)+1}\right. \\
\left.+v_{j k}^{-\tilde{\sigma}_{j k}\left(\gamma_{D}+2\right)}|t|^{-\left(2-\tilde{\sigma}_{j k}\right)\left(\gamma_{D}+2\right)+1}\right) .
\end{gathered}
$$

By a straightforward calculation, provided $\tilde{\sigma}_{j k}<2-\left(1+\theta_{j k}\right) /\left(\gamma_{D}+\mu\right)$,

$$
\int d t I_{11}=O\left(v_{j k}^{-b}\right)
$$

having taken

$$
b=\frac{\tilde{\sigma}_{j k}}{2-\tilde{\sigma}_{j k}}=\min \left\{\frac{\tilde{\sigma}_{j k}}{2-\tilde{\sigma}_{j k}}, \frac{\tilde{\sigma}_{j k}+\left(2-\gamma_{1} / 2-\theta_{j k}\right) /\left(\gamma_{D}+1\right)}{2-\tilde{\sigma}_{j k}-\theta_{j k} /\left(\gamma_{D}+1\right)}, \frac{\tilde{\sigma}_{j k}+\left(2-\gamma_{1} / 2-\theta_{j k}\right) /\left(\gamma_{D}+\mu\right)}{2-\tilde{\sigma}_{j k}-\theta_{j k} /\left(\gamma_{D}+\mu\right)}\right\} .
$$


Using Adachi and Maehara's computations [2] of the last three terms of the integral of $I_{3}$ in the proof of their Lemma 3.4, assuming that $\tilde{\sigma}_{j k}<2-2 /\left(\gamma_{D}+2 \mu\right)$, we obtain:

$$
\int_{-\infty}^{+\infty} d t I_{12}=O\left(v_{j k}^{-\tilde{\sigma}_{j k} /\left[\left(2-\tilde{\sigma}_{j k}\right)-1 /\left(\gamma_{D}+2\right)\right]}\right) .
$$

Thus we have, in general:

$$
\int_{-\infty}^{+\infty} d t I_{1}=O\left(v_{j k}^{-\sigma_{j k}}\right)
$$

If $|\mathbf{x}| \leq(5 / 8) v_{j k}^{\sigma_{j k}}|t|$ and $v_{j k}^{\sigma_{j k}-1} \leq(2 / 5) \sqrt{1-\delta_{j k}}$, for $q_{j k} \neq 0$, we obtain as in (2.33)

$$
\left|\mathbf{x}+\mathbf{v}_{j k} t+\mathbf{e}_{1} q_{j k} E t^{2} /\left(2 \mu_{j k}\right)\right| \geq c v_{j k}^{\tilde{\sigma}_{j k}}|t|^{2-\tilde{\sigma}_{j k}},
$$

by equations (1.31)-(1.34) and (2.36) we can invoke Proposition 2.10 from Enss [14] in (2.31) to estimate $I_{2}$ with $v_{0}>4 \eta_{j k}$,

$$
\begin{aligned}
I_{2} & \leq C\left\|\left(V_{j k}^{l}-V_{j k, v_{j k} t}^{l}\right)\left(\mathbf{x}+\mathbf{v}_{j k} t+\mathbf{e}_{1} q_{j k} E t^{2} /\left(2 \mu_{j k}\right)\right) e^{-i t \mathbf{p}^{2} /\left(2 \mu_{j k}\right)} g(\mathbf{p}) F\left(|\mathbf{x}|<v_{j k}^{\sigma_{j k}}|t| / 8\right)\right\| \\
& \leq C \| F\left(\left|\mathbf{x}-\mathbf{v}_{j k} t\right| \geq\left\{\begin{array}{ll}
5 v_{j k}^{\sigma_{j k}}|t| / 8, & \text { if } q_{j k} \neq 0, \\
v_{j k}|t| / 2, & \text { if } q_{j k}=0,
\end{array}\right) e^{-i t \mathbf{p}^{2} /\left(2 \mu_{j k}\right)} g\left(\mathbf{p}-\mu_{j k} \mathbf{v}_{j k}\right) F\left(|\mathbf{x}|<v_{j k}^{\sigma_{j k}}|t| / 8\right) \|\right. \\
& \leq C\left(1+v_{j k}^{\sigma_{j k}}|t|\right)^{-2} .
\end{aligned}
$$

Again, by Lemma 2.2 equation (2.17), we estimate $I_{3}$,

$$
I_{3} \leq C\left(1+v_{j k}^{\sigma_{j k}}|t|\right)^{-2} .
$$

By (2.35), (2.37) and (2.38) we finish the proof.

Let us denote,

$$
I_{G, v, a, b}=\exp \left(-i \sum_{j<k}^{E} \int_{a}^{b} d s V_{j k}^{E, s}\left(\mathbf{v}_{j k} s+\mathbf{e}_{1} q_{j k} E s^{2} /\left(2 \mu_{j k}\right)\right)\right) \text { and } I_{G, v}=I_{G, v,-\infty, \infty} .
$$

Observe that $\tilde{U}_{G, v}(t)=I_{G, v, 0, t}$.

PROPOSITION 2.6. The wave operators $\Omega_{ \pm}^{D, G, v}$ and $\Omega_{ \pm}^{G, v}$ exist and, moreover,

$$
\Omega_{ \pm}^{D, G, v}=W_{ \pm}^{D} I_{G, v, 0, \pm \infty}, \quad \Omega_{ \pm}^{G, v}=W_{ \pm} I_{G, v, 0, \pm \infty} .
$$

Proof. We give only the proof for $\Omega_{ \pm}^{D, G, v}$, the other is similar. Note that:

$$
\mathrm{s}-\lim _{t \rightarrow \pm \infty} e^{i t H} U^{D, G, v}(t)=\mathrm{s}-\lim _{t \rightarrow \pm \infty} e^{i t H} e^{-i t H_{0}} \tilde{U}_{D}(t) I_{G, v, 0, t}=W_{ \pm}^{D} \mathrm{~s}-\lim _{t \rightarrow \pm \infty} I_{G, v, 0, t} .
$$

Furthermore, for any $\Phi \in L^{2}$ we have that:

$$
\left\|\left(I_{G, v, 0, t}-I_{G, v, 0, \pm \infty}\right) \Phi\right\|^{2}=\int_{\mathbb{R}^{n}}\left|I_{G, v, 0, t}-I_{G, v, 0, \pm \infty}\right|^{2}|\Phi|^{2} \longrightarrow_{t \rightarrow \pm \infty} 0,
$$

by the Lebesgue dominated convergence theorem, taking into account that the integrand is dominated by $4|\Phi|^{2}$ for all $t$. This proves the proposition.

Now we focus in the wave operator estimates. We use Jacobi Coordinates based on the pair $(1,2)$, where $v=|\mathbf{v}|=\left|\mathbf{v}_{2}-\mathbf{v}_{1}\right|$ and $v_{j k}=O\left(v^{2}\right)$, for $(j, k) \neq(1,2)$. Lemma 2.7. below, is a N-body generalization of Lemma 3.5 in Adachi and Maehara 2. See also Lemma 4.6 of Adachi, Kamada, Kazuno and Toratani [1, for a generalization of Lemma 3.5 of Adachi and Maehara [2] to the case where the external electric field is asymptotically zero in time, in the two-body case. 
LEMMA 2.7. Let $\alpha$ be as in Definition 1.2, where, without loss of generality, $\alpha=1$ if $q_{j k}=0$ for all $1 \leq j<k \leq N$. For all $1 \leq j<k \leq N$, let $0<\sigma_{j k} \leq 1$ be as in Lemma 2.5. Let us take $V^{V S} \in \mathcal{V}_{V S R}, V^{S} \in$ $\mathcal{V}_{S R}, V^{L} \in \mathcal{V}_{L R}$. Then, for all $\Phi_{\mathbf{v}}$ as in (1.13) with a fixed normalized $\hat{\phi}_{3}$, where, with $\delta_{j k}$ being defined as in (2.12), the relative velocities satisfy $\left|\hat{\mathbf{v}}_{j k} \cdot \hat{\mathbf{E}}\right| \leq \delta_{j k}$ for all integers $1 \leq j<k \leq N$ with $q_{j, k} \neq 0$, and $v_{j k}>v_{0}^{1 / \sigma_{j k}}$ for some $v_{0}>0$ and all integers $1 \leq j<k \leq N$ :

$$
\sup _{t \in \mathbb{R}}\left\|\left(\Omega_{ \pm}^{D, G, v}-e^{i t H} U^{D, G, v}(t)\right) \Phi_{\mathbf{v}}\right\|=O\left(v^{-\min \left\{\alpha, \sigma_{j k} \mid 1 \leq j<k \leq N\right\}}\right) .
$$

In the short-range case, where $V_{j k}^{l}=0$ (see (2.1)) for all $1 \leq j<k \leq N$, we obtain the following result

$$
\sup _{t \in \mathbb{R}}\left\|\left(\Omega_{ \pm}^{G, v}-e^{i t H} U^{G, v}(t)\right) \Phi_{\mathbf{v}}\right\|= \begin{cases}O\left(v^{-\alpha}\right), & \text { if } \alpha<1 \text { and } \sum_{j<k}\left|q_{j k}\right|>0, \\ O\left(v^{-\left(1-\epsilon_{1}\right)}\right), & \text { if } \alpha=1 \text { and } \sum_{j<k}\left|q_{j k}\right|>0 \\ O\left(v^{-1}\right), & \text { if } \sum_{j<k}\left|q_{j k}\right|=0,\end{cases}
$$

for all $0<\epsilon_{1}<1$.

Proof. We give the proof for $\Omega_{+}^{D, G, v}$. By Duhamel's formula, (1.31) and (1.33):

$$
\begin{aligned}
\Omega_{+}^{D, G, v}-e^{i t H} U^{D, G, v}(t)= & \lim _{t^{\prime} \rightarrow+\infty} e^{i t^{\prime} H} U^{D, G, v}\left(t^{\prime}\right)-e^{i t H} U^{D, G, v}(t)=\lim _{t^{\prime} \rightarrow+\infty} \int_{t}^{t^{\prime}} d s \frac{d}{d s}\left(e^{i s H} U^{D, G, v}(s)\right) \\
= & i \int_{t}^{\infty} d s e^{i s H}\left(\sum _ { j < k } \left[V_{j k}^{v s}\left(\tilde{\mathbf{x}}_{k}-\tilde{\mathbf{x}}_{j}\right)+V_{j k}^{l}\left(\tilde{\mathbf{x}}_{k}-\tilde{\mathbf{x}}_{j}\right)\right.\right. \\
& \left.-V_{j k}^{l}\left(s \mathbf{p}_{j k} / \mu_{j k}-\mathbf{e}_{1} q_{j k} E s^{2} /\left(2 \mu_{j k}\right)\right)\right] \\
& \left.+\sum_{j<k}^{E}\left[V_{j k}^{E, s}\left(\tilde{\mathbf{x}}_{k}-\tilde{\mathbf{x}}_{j}\right)-V_{j k}^{E, s}\left(\mathbf{v}_{j k} s+\mathbf{e}_{1} q_{j k} E s^{2} /\left(2 \mu_{j k}\right)\right)\right]\right) U^{D, G, v}(s) .
\end{aligned}
$$

Using that the Graf-type modifier $\tilde{U}_{G, v}(t)(2.2)$ conmutes with any operator, and Lemmata 2.3, 2.4, 2.5, it follows for any $0<\epsilon_{1}<1, t \in \mathbb{R}$ :

$$
\begin{aligned}
& \left\|\left(e^{-i t H} \Omega_{+}^{D, G, v}-U^{D, G, v}(t)\right) \Phi_{\mathbf{v}}\right\| \leq C \sum_{j<k} \int_{-\infty}^{\infty} \| V_{j k}^{v s}\left(\tilde{\mathbf{x}}_{k}-\tilde{\mathbf{x}}_{j}\right) \\
& \times e^{-i s H_{0}} \tilde{U}_{D}(s) \prod_{j^{\prime}<k^{\prime}} f_{j^{\prime} k^{\prime}}\left(\mathbf{p}_{j^{\prime} k^{\prime}}-\mu_{j^{\prime} k^{\prime}} \mathbf{v}_{j^{\prime} k^{\prime}}\right)\left(1+\left|\tilde{\mathbf{x}}_{k}-\tilde{\mathbf{x}}_{j}\right|^{2}\right)^{-2} \| d s \\
& +C \sum_{j<k} \int_{-\infty}^{\infty} \|\left(V_{j k}^{l}\left(\tilde{\mathbf{x}}_{k}-\tilde{\mathbf{x}}_{j}\right)-V_{j k}^{l}\left(s \mathbf{p}_{j k} / \mu_{j k}-\mathbf{e}_{1} q_{j k} E s^{2} /\left(2 \mu_{j k}\right)\right)\right) \\
& \times e^{-i s H_{0}} \tilde{U}_{D}(s) \prod_{j^{\prime}<k^{\prime}} f_{j^{\prime} k^{\prime}}\left(\mathbf{p}_{j^{\prime} k^{\prime}}-\mu_{j^{\prime} k^{\prime}} \mathbf{v}_{j^{\prime} k^{\prime}}\right)\left(1+\left|\tilde{\mathbf{x}}_{k}-\tilde{\mathbf{x}}_{j}\right|^{2}\right)^{-2} \| d s \\
& +C \sum_{j<k}^{E} \int_{-\infty}^{\infty} \|\left(V_{j k}^{s}\left(\tilde{\mathbf{x}}_{k}-\tilde{\mathbf{x}}_{j}\right)-V_{j k}^{s}\left(\mathbf{v}_{j k} s+\mathbf{e}_{1} q_{j k} E s^{2} /\left(2 \mu_{j k}\right)\right)\right) \\
& \times e^{-i s H_{0}} \tilde{U}_{D}(s) \prod_{j^{\prime}<k^{\prime}} f_{j^{\prime} k^{\prime}}\left(\mathbf{p}_{j^{\prime} k^{\prime}}-\mu_{j^{\prime} k^{\prime}} \mathbf{v}_{j^{\prime} k^{\prime}}\right)\left(1+\left|\tilde{\mathbf{x}}_{k}-\tilde{\mathbf{x}}_{j}\right|^{2}\right)^{-2} \| d s \\
& \leq \sum_{j<k}\left(O\left(v_{j k}^{-1}\right)+O\left(v_{j k}^{-\sigma_{j k}}\right)\right)+\sum_{j<k}^{E} \begin{cases}O\left(v_{j k}^{-\alpha}\right), & \text { if } \alpha<1, \\
O\left(v_{j k}^{-\left(1-\epsilon_{1}\right)}\right), & \text { if } \alpha=1 .\end{cases}
\end{aligned}
$$


The proof is finished by the use of the following arguments: $\alpha<1$ implies for $v \geq 1$ that $v^{-1} \leq v^{-\alpha}$, $0<\sigma_{j k}<1$ implies, for $\epsilon_{1}$ sufficiently small that $O\left(v_{j k}^{-\left(1-\epsilon_{1}\right)}\right) \leq O\left(v_{j k}^{-\sigma_{j k}}\right)$, and noting that $v_{12}=v$ and $v_{j k}$ is $O\left(v^{2}\right)$ for $j<k=3, \ldots, N$.

Lemma 2.5 above defines two sets of exponents $\sigma_{j k}$ and $\tilde{\sigma}_{j k}$. Theorem 2.8 below needs $\sigma_{j k}>1 / 2$. For this purpose we have to ask, for all $1 \leq j<k \leq N$ with $q_{j k} \neq 0$ and $V_{j k}^{l} \neq 0$, that $\theta_{j k}$, being as in (2.15), must hold:

$$
2-\max \left\{\frac{1+\theta_{j k}}{\gamma_{D}+\mu}, \frac{2}{\gamma_{D}+2 \mu}, 1\right\}>\frac{2}{3} \Longleftrightarrow \theta_{j k}<\frac{4}{3}\left(\gamma_{D}+\mu\right)-1 .
$$

In particular, inequality (2.43) is always true if $\theta_{j k} \leq 1 / 3$ because $1 / 3<4\left(\gamma_{D}+\mu\right) / 3-1$ for all $\gamma_{D}$ and $\mu$ as in Definition 1.2. Inequality (2.43) is always met in conditions $\zeta_{j k}^{b}$ and $\zeta_{j k}^{c}$, see (2.15), because in the former, $\theta_{j k}$ can be taken arbitrarily small, and in the later, $\theta_{j k}$ is zero. If there is a pair $(j, k)$ with $q_{j k} \neq 0$ and the condition $\zeta_{j k}^{a}$ is true, (2.43) is equivalent to $\max \left\{3 / 2,3-4\left(\gamma_{D}+\mu\right) / 3\right\}<\gamma_{1}<2$. If $\sum\left|q_{j k}\right|=0$ we just need $3 / 2<\gamma_{1} \leq 2$. Theorem 1.3 is stated considering long-range potentials, in this case, $\zeta_{j k}^{a}$ is true for some pair $(j, k)$ with $q_{j k} \neq 0$, if and only if, there are two pairs $\left(j^{*}, k^{*}\right)$ and $\left(j^{\prime}, k^{\prime}\right)$ such that $1 \leq j^{*}<k^{*} \leq N$, $1 \leq j^{\prime}<k^{\prime} \leq N, q_{j^{*} k^{*}} \neq 0$ and $q_{j^{\prime} k^{\prime}}=0$. We can also use Theorem 1.3 with short-range potentials: the condition $3-4\left(\gamma_{D}+\mu\right) / 3<\gamma_{1}$ is always true because, without loss of generality, we can take $\gamma_{1}=2$ in this situation.

THEOREM 2.8. (Reconstruction Formula) Let $\gamma_{1}$ be as in Definition 1.1, $\alpha, \gamma_{D}, \mu$ be as in Definition 1.2, where, without loss of generality, $\alpha=1$ if $q_{j k}=0$ for all $1 \leq j<k \leq N$. If there exists two pairs $1 \leq j<k \leq N, 1 \leq j^{\prime}<k^{\prime} \leq N$ such that $q_{j k} \neq 0, q_{j^{\prime} k^{\prime}}=0, V_{j^{\prime} k^{\prime}}^{l} \neq 0$, and either $j^{\prime}=j$ or $j^{\prime}=$ $k$ or $k^{\prime}=j$ or $k^{\prime}=k$ or $j^{\prime}+j=3$, we additionally assume $\gamma_{1}>3-4\left(\gamma_{D}+\mu\right) / 3$. For all $1 \leq j<k \leq N$, let $0<\sigma_{j k} \leq 1$ be as in Lemma 2.5. Let us take $V^{V S} \in \mathcal{V}_{V S R}, V^{S} \in \mathcal{V}_{S R}, V^{L} \in \mathcal{V}_{L R}$, where $V_{12}^{\text {vs }}$ satisfies (2.21) for all $g \in C_{0}^{\infty}\left(\mathbb{R}^{n}\right)$, with $0 \leq \rho \leq 2 \min \left\{\alpha, \sigma_{j k} \mid 1 \leq j<k \leq N\right\}-1$. Let us set $\mathbf{p}_{l}=\mathbf{p} \cdot \mathbf{e}_{l}$ for any $l=1, \ldots, N$. Then, for all $\Phi_{\mathbf{v}}, \Psi_{\mathbf{v}}$ as in (1.13) with the same fixed normalized $\hat{\phi}_{3}$, with $\delta_{j k}$ being defined as in (2.12), the relative velocities satisfy $\left|\hat{\mathbf{v}}_{j k} \cdot \hat{\mathbf{E}}\right| \leq \delta_{j k}$ for all integers $1 \leq j<k \leq N$ with $q_{j, k} \neq 0$, and $v_{j k}>v_{0}^{1 / \sigma_{j k}}$ for some $v_{0}>0$, as in Lemma 2.5, and all integers $1 \leq j<k \leq N$ :

$$
\begin{aligned}
v\left(i\left[S^{D}, \mathbf{p}_{l}\right] \Phi_{\mathbf{v}}, \Psi_{\mathbf{v}}\right)= & \int_{-\infty}^{\infty} d \tau\left[\left(V_{12}^{v s}(\mathbf{x}+\tau \hat{\mathbf{v}}) \mathbf{p}_{l} \Phi_{12}, \Psi_{12}\right)-\left(V_{12}^{v s}(\mathbf{x}+\tau \hat{\mathbf{v}}) \Phi_{12}, \mathbf{p}_{l} \Psi_{12}\right)\right. \\
+ & \left.i\left(\left(\frac{\partial V_{12}^{s}}{\partial x_{l}}\right)(\mathbf{x}+\tau \hat{\mathbf{v}}) \Phi_{12}, \Psi_{12}\right)+i\left(\left(\frac{\partial V_{12}^{l}}{\partial x_{l}}\right)(\mathbf{x}+\tau \hat{\mathbf{v}}) \Phi_{12}, \Psi_{12}\right)\right] \\
+ & \left\{\begin{aligned}
o\left(v^{-\rho_{l}}\right), & \text { if } \gamma_{2}-1 \leq \rho \leq 2 \min \left\{\alpha, \sigma_{j k} \mid 1 \leq j<k \leq N\right\}-1<1, \\
& \text { for any } \rho_{l}, 0 \leq \rho_{l}<\gamma_{2}-1, \\
o\left(v^{-\rho}\right), & \text { if } 0 \leq \rho<\min \left\{\gamma_{2}, 2 \alpha, 2 \sigma_{j k} \mid 1 \leq j<k \leq N\right\}-1, \\
O\left(v^{-\rho}\right), & \text { if } \rho=2 \min \left\{\alpha, \sigma_{j k} \mid 1 \leq j<k \leq N\right\}-1<\gamma_{2}-1, \\
o\left(v^{-\rho_{l}}\right), & \text { if } \rho=1, \sum\left|q_{j k}\right|=0 \text { and } V_{12}^{l} \neq 0, \\
O\left(v^{-1}\right), & \text { if } \rho=1, \sum\left|q_{j k}\right|=0 \text { and } V_{12}^{l}=0 .
\end{aligned}\right.
\end{aligned}
$$

$$
\text { where } \gamma_{2}:= \begin{cases}\gamma_{1}, & \text { if } q_{12}=0 \text { and } V_{12}^{l} \neq 0, \\ \gamma_{D}+\mu, & \text { if } q_{12} \neq 0, \text { and } V_{12}^{l} \neq 0, \\ 2, & \text { if } V_{12}^{l}=0 .\end{cases}
$$

REMARK 2.9. Note that the first term in the right-hand side of (2.44) can be written as

$$
i \int_{-\infty}^{\infty} d \tau\left(\left(\frac{\partial V_{12}}{\partial x_{l}}\right)(x+\tau \hat{v}) \Phi_{12}, \Psi_{12}\right)
$$


where $V_{12}=V_{12}^{v s}+V_{12}^{s}+V_{12}^{l}$, and the derivative $\frac{\partial V_{12}}{\partial x_{l}}$ is taken in distribution sense. This shows that the high-velocity limit of $v\left(i\left[S^{D}, \mathbf{p}_{l}\right] \Phi_{\mathbf{v}}, \Psi_{\mathbf{v}}\right)$ is independent of the decomposition of the potential $V$ into the part $V^{V S}+V^{S}$, that is of short range under the constant electric field $\mathbf{E}$ and the part $V^{L}$ that is long-range; that is used for the definition of the Dollard scattering operator (2.10).

Proof.

The scattering operator can be expressed as $S^{D}=\left(\Omega_{+}^{D, G, v} I_{G, v, 0,+\infty}^{-1}\right)^{*}\left(\Omega_{-}^{D, G, v} I_{G, v, 0,-\infty}^{-1}\right)=$ $I_{G, v}\left(\Omega_{+}^{D, G, v}\right)^{*} \Omega_{-}^{D, G, v}$, by (2.10) and (2.40). Noting that $\left[S^{D}, \mathbf{p}_{l}\right]=\left[S^{D}, \mathbf{p}_{l}-\mu_{12} v_{l}\right]=\left[S^{D}-I_{G, v}, \mathbf{p}_{l}-\mu_{12} v_{l}\right]$ and $\left(\mathbf{p}_{l}-\mu_{12} v_{l}\right) \Phi_{\mathbf{v}}=\left(\mathbf{p}_{l} \Phi_{0}\right)_{\mathbf{v}}$ where $\mathbf{p}_{l}$ and $v_{l}$ are the l-th components of the relative momentum and the velocity $\mathbf{v}$ of the chosen pair $(1,2)$, respectively. Since $\Omega_{ \pm}^{D, G, v}$ are partially isometric and, by Duhamel formula, (1.31) and (1.33), as in the proof of Lemma 2.7

$$
i\left(S^{D}-I_{G, v}\right) \Phi_{\mathbf{v}}=I_{G, v} i\left(\Omega_{+}^{D, G, v}-\Omega_{-}^{D, G, v}\right)^{*} \Omega_{-}^{D, G, v} \Phi_{\mathbf{v}}=I_{G, v} \int_{-\infty}^{+\infty} d t\left(U^{D, G, v}(t)\right)^{*} V_{t}(\tilde{\mathbf{x}}) e^{-i H t} \Omega_{-}^{D, G, v} \Phi_{\mathbf{v}},
$$

with $\tilde{\mathbf{x}}$ defined as (1.2) and $V_{t}=V_{3, t}+V_{12, t}$ where

$$
\begin{aligned}
V_{3, t}(\tilde{\mathbf{x}})= & \sum_{j<k, 3 \leq k \leq N}\left[V_{j k}^{v s}\left(\tilde{\mathbf{x}}_{k}-\tilde{\mathbf{x}}_{j}\right)+V_{j k}^{l}\left(\tilde{\mathbf{x}}_{k}-\tilde{\mathbf{x}}_{j}\right)-V_{j k}^{l}\left(t \mathbf{p}_{j k} / \mu_{j k}-\mathbf{e}_{1} q_{j k} E t^{2} /\left(2 \mu_{j k}\right)\right)\right] \\
& +\sum_{j<k, 3 \leq k \leq N}^{E}\left[V_{j k}^{s}\left(\tilde{\mathbf{x}}_{k}-\tilde{\mathbf{x}}_{j}\right)-V_{j k}^{s}\left(\mathbf{v}_{j k} t+\mathbf{e}_{1} q_{j k} E t^{2} /\left(2 \mu_{j k}\right)\right)\right]
\end{aligned}
$$

and

$$
\begin{aligned}
V_{12, t}= & V_{12}^{v s}(\mathbf{x})+V_{12}^{l}(\mathbf{x})-V_{12}^{l}\left(t \mathbf{p} / \mu_{12}-\mathbf{e}_{1} q_{12} E t^{2} /\left(2 \mu_{12}\right)\right) \\
& +V_{12}^{s}(\mathbf{x})-V_{12}^{s}\left(\mathbf{v} t+\mathbf{e}_{1} q_{12} E t^{2} /\left(2 \mu_{12}\right)\right) .
\end{aligned}
$$

Thus we have

$$
v\left(i\left[S^{D}, \mathbf{p}_{l}\right] \Phi_{\mathbf{v}}, \Psi_{\mathbf{v}}\right)=I_{G, v}(I(v)+R(v))
$$

with

$$
I(v)=\int_{-\infty}^{+\infty} d \tau l_{v}(\tau)
$$

where

$$
\begin{aligned}
l_{v}(v t)= & \left(V_{12, t}(\mathbf{x}) e^{-i t H_{0}} \tilde{U}_{D}(t)\left(\mathbf{p}_{l} \Phi_{0}\right)_{\mathbf{v}}, e^{-i t H_{0}} \tilde{U}_{D}(t) \Psi_{\mathbf{v}}\right) \\
& -\left(V_{12, t}(\mathbf{x}) e^{-i t H_{0}} \tilde{U}_{D}(t) \Phi_{\mathbf{v}}, e^{-i t H_{0}} \tilde{U}_{D}(t)\left(\mathbf{p}_{l} \Psi_{0}\right)_{\mathbf{v}}\right)
\end{aligned}
$$

and

$$
\begin{aligned}
R(v) / v= & \int_{-\infty}^{+\infty} d t\left[\left(V_{3, t} e^{-i t H_{0}} \tilde{U}_{D}(t)\left(\mathbf{p}_{l} \Phi_{0}\right)_{\mathbf{v}}, e^{-i t H_{0}} \tilde{U}_{D}(t) \Psi_{\mathbf{v}}\right)\right. \\
& -\left(V_{3, t} e^{-i t H_{0}} \tilde{U}_{D}(t) \Phi_{\mathbf{v}}, e^{-i t H_{0}} \tilde{U}_{D}(t)\left(\mathbf{p}_{l} \Psi_{0}\right)_{\mathbf{v}}\right)+\left(\left(e^{-i H t} \Omega_{-}^{D, G, v}-U^{D, G, v}(t)\right)\left(\mathbf{p}_{l} \Phi_{0}\right)_{\mathbf{v}},\right. \\
& \left.\left.\quad V_{t} U^{D, G, v}(t) \Psi_{\mathbf{v}}\right)-\left(\left(e^{-i H t} \Omega_{-}^{D, G, v}-U^{D, G, v}(t)\right) \Phi_{\mathbf{v}}, V_{t} U^{D, G, v}(t)\left(\mathbf{p}_{l} \Psi_{0}\right)_{\mathbf{v}}\right)\right] .
\end{aligned}
$$

In the derivation of (2.48) and (2.49) we used that $\tilde{U}_{G, v}(t)$ commutes with any operator.

We are going to need to translate the Dollard-type modifier (2.3)

$$
\tilde{U}_{D}(\mathbf{v}, t)=e^{-i \mu_{12} \mathbf{v} \cdot \mathbf{x}} \prod_{j=3}^{N} e^{-i \mu_{j} \mathbf{v}_{j} \cdot \mathbf{x}_{j}} \tilde{U}_{D}(t) e^{i \mu_{12} \mathbf{v} \cdot \mathbf{x}} \prod_{j=3}^{N} e^{i \mu_{j} \mathbf{v}_{j} \cdot \mathbf{x}_{j}} .
$$


Using equations (1.31)-(1.35) and substituting (2.45) and (2.50) in (2.48), it follows that

$$
I(v)=J_{1}(v)-J_{2}(v)+i J_{3}(v)+i J_{4}(v)
$$

where

$$
\begin{aligned}
J_{1}(v)= & \int\left(V_{12}^{v s}\left(\mathbf{x}+\tau \hat{\mathbf{v}}+\mathbf{e}_{1} q_{12} E \tau^{2} /\left(2 v^{2} \mu_{12}\right)\right) e^{-i \tau \mathbf{p}^{2} /\left(2 v \mu_{12}\right)} \tilde{U}_{D}(\mathbf{v}, \tau / v) \mathbf{p}_{l} \Phi_{0},\right. \\
& \left.e^{-i \tau \mathbf{p}^{2} /\left(2 v \mu_{12}\right)} \tilde{U}_{D}(\mathbf{v}, \tau / v) \Psi_{0}\right) d \tau \\
J_{2}(v)= & \int\left(V_{12}^{v s}\left(\mathbf{x}+\tau \hat{\mathbf{v}}+\mathbf{e}_{1} q_{12} E \tau^{2} /\left(2 v^{2} \mu_{12}\right)\right) e^{-i \tau \mathbf{p}^{2} /\left(2 v \mu_{12}\right)} \tilde{U}_{D}(\mathbf{v}, \tau / v) \Phi_{0}\right. \\
& \left.e^{-i \tau \mathbf{p}^{2} /\left(2 v \mu_{12}\right)} \tilde{U}_{D}(\mathbf{v}, \tau / v) \mathbf{p}_{l} \Psi_{0}\right) d \tau \\
J_{3}(v)= & \int\left(\left(\partial V_{12}^{E, s} / \partial x_{l}\right)\left(\mathbf{x}+\tau \hat{\mathbf{v}}+\mathbf{e}_{1} q_{12} E \tau^{2} /\left(2 v^{2} \mu_{12}\right)\right) e^{-i \tau \mathbf{p}^{2} /\left(2 v \mu_{12}\right)} \tilde{U}_{D}(\mathbf{v}, \tau / v) \Phi_{0},\right. \\
& \left.e^{-i \tau \mathbf{p}^{2} /\left(2 v \mu_{12}\right)} \tilde{U}_{D}(\mathbf{v}, \tau / v) \Psi_{0}\right) d \tau . \\
J_{4}(v)= & \int\left(\left(\partial V_{12}^{l} / \partial x_{l}\right)\left(\mathbf{x}+\tau \hat{\mathbf{v}}-\mathbf{e}_{1} q_{12} E \tau^{2} /\left(2 v^{2} \mu_{12}\right)\right) e^{-i \tau \mathbf{p}^{2} /\left(2 v \mu_{12}\right)} \tilde{U}_{D}(\mathbf{v}, \tau / v) \Phi_{0},\right. \\
& \left.e^{-i \tau \mathbf{p}^{2} /\left(2 v \mu_{12}\right)} \tilde{U}_{D}(\mathbf{v}, \tau / v) \mathbf{p}_{l} \Psi_{0}\right) d \tau .
\end{aligned}
$$

There exists $C>0$ that uniformly bounds the following expression, for all $j<k$ :

$$
\left\|\Phi_{\mathbf{v}}\right\|+\left\|\left(\mathbf{p}_{l} \Phi_{0}\right)_{\mathbf{v}}\right\|+\left\|\left(1+\left|\tilde{\mathbf{x}}_{k}-\tilde{\mathbf{x}}_{j}\right|^{2}\right)^{2} \Phi_{\mathbf{v}}\right\|+\left\|\left(1+\left|\tilde{\mathbf{x}}_{k}-\tilde{\mathbf{x}}_{j}\right|^{2}\right)^{2}\left(\mathbf{p}_{l} \Phi_{0}\right)_{\mathbf{v}}\right\| \leq C
$$

Then,

$$
\begin{aligned}
& \frac{|R(v)|}{v} \leq C \sum_{j<k, 3 \leq k \leq N} \int d t\left\|V_{j k}^{v s}\left(\tilde{\mathbf{x}}_{k}-\tilde{\mathbf{x}}_{j}\right) e^{-i t H_{0}} \tilde{U}_{D}(t) \prod_{j^{\prime}<k^{\prime}} f_{j^{\prime} k^{\prime}}\left(\mathbf{p}_{j^{\prime} k^{\prime}}-\mu_{j^{\prime} k^{\prime} \mathbf{v}_{j^{\prime} k^{\prime}}}\right)\left(1+\left|\tilde{\mathbf{x}}_{k}-\tilde{\mathbf{x}}_{j}\right|^{2}\right)^{-2}\right\| \\
& +C \sum_{j<k, 3 \leq k \leq N} \int d t \|\left(V_{j k}^{l}\left(\tilde{\mathbf{x}}_{k}-\tilde{\mathbf{x}}_{j}\right)-V_{j k}^{l}\left(t \mathbf{p}_{j k} / \mu_{j k}-\mathbf{e}_{1} q_{j k} E t^{2} /\left(2 \mu_{j k}\right)\right)\right) \\
& \times e^{-i t H_{0}} \tilde{U}_{D}(t) \prod_{j^{\prime}<k^{\prime}} f_{j^{\prime} k^{\prime}}\left(\mathbf{p}_{j^{\prime} k^{\prime}}-\mu_{j^{\prime} k^{\prime}} \mathbf{v}_{j^{\prime} k^{\prime}}\right)\left(1+\left|\tilde{\mathbf{x}}_{k}-\tilde{\mathbf{x}}_{j}\right|^{2}\right)^{-2} \| \\
& +C \sum_{j<k, 3 \leq k \leq N}^{E} \int d t \|\left(V_{j k}^{s}\left(\tilde{\mathbf{x}}_{k}-\tilde{\mathbf{x}}_{j}\right)-V_{j k}^{s}\left(\mathbf{v}_{j k} t+\mathbf{e}_{1} q_{j k} E t^{2} /\left(2 \mu_{j k}\right)\right)\right) \\
& \times e^{-i t H_{0}} \tilde{U}_{D}(t) \prod_{j^{\prime}<k^{\prime}} f_{j^{\prime} k^{\prime}}\left(\mathbf{p}_{j^{\prime} k^{\prime}}-\mu_{j^{\prime} k^{\prime} \mathbf{v}_{j^{\prime} k^{\prime}}}\right)\left(1+\left|\tilde{\mathbf{x}}_{k}-\tilde{\mathbf{x}}_{j}\right|^{2}\right)^{-2} \| \\
& +C\left[\sup _{t \in \mathbb{R}}\left\|\left(e^{-i H t} \Omega_{-}^{D, G, v}-U^{D, G, v}(t)\right)\left(\mathbf{p}_{l} \Phi_{0}\right)_{\mathbf{v}}\right\|+\sup _{t \in \mathbb{R}}\left\|\left(e^{-i H t} \Omega_{-}^{D, G, v}-U^{D, G, v}(t)\right) \Phi_{\mathbf{v}}\right\|\right] \\
& \times\left[\sum_{j<k} \int d t\left\|V_{j k}^{v s}\left(\tilde{\mathbf{x}}_{k}-\tilde{\mathbf{x}}_{j}\right) e^{-i t H_{0}} \tilde{U}_{D}(t) \prod_{j^{\prime}<k^{\prime}} f_{j^{\prime} k^{\prime}}\left(\mathbf{p}_{j^{\prime} k^{\prime}}-\mu_{j^{\prime} k^{\prime}} \mathbf{v}_{j^{\prime} k^{\prime}}\right)\left(1+\left|\tilde{\mathbf{x}}_{k}-\tilde{\mathbf{x}}_{j}\right|^{2}\right)^{-2}\right\|\right.
\end{aligned}
$$




$$
\begin{aligned}
& +\sum_{j<k} \int d t \|\left(V_{j k}^{l}\left(\tilde{\mathbf{x}}_{k}-\tilde{\mathbf{x}}_{j}\right)-V_{j k}^{l}\left(t \mathbf{p}_{j k} / \mu_{j k}-\mathbf{e}_{1} q_{j k} E t^{2} /\left(2 \mu_{j k}\right)\right)\right) \\
& \times e^{-i t H_{0}} \tilde{U}_{D}(t) \prod_{j^{\prime}<k^{\prime}} f_{j^{\prime} k^{\prime}}\left(\mathbf{p}_{j^{\prime} k^{\prime}}-\mu_{j^{\prime} k^{\prime}} \mathbf{v}_{j^{\prime} k^{\prime}}\right)\left(1+\left|\tilde{\mathbf{x}}_{k}-\tilde{\mathbf{x}}_{j}\right|^{2}\right)^{-2} \| \\
& +\sum_{j<k}^{E} \int d t \|\left(V_{j k}^{s}\left(\tilde{\mathbf{x}}_{k}-\tilde{\mathbf{x}}_{j}\right)-V_{j k}^{s}\left(\mathbf{v}_{j k} t+\mathbf{e}_{1} q_{j k} E t^{2} /\left(2 \mu_{j k}\right)\right)\right) \\
& \left.\times e^{-i t H_{0}} \tilde{U}_{D}(t) \prod_{j^{\prime}<k^{\prime}} f_{j^{\prime} k^{\prime}}\left(\mathbf{p}_{j^{\prime} k^{\prime}}-\mu_{j^{\prime} k^{\prime}} \mathbf{v}_{j^{\prime} k^{\prime}}\right)\left(1+\left|\tilde{\mathbf{x}}_{k}-\tilde{\mathbf{x}}_{j}\right|^{2}\right)^{-2} \|\right] .
\end{aligned}
$$

Thus, by Lemmata 2.3, 2.4 and 2.7, if $V_{j k}^{l}=0$ for all $1 \leq j<k \leq N$ :

$$
R(v)= \begin{cases}o\left(v^{-\rho}\right), & \text { if } 0 \leq \rho<2 \alpha-1, \\ O\left(v^{-\rho}\right), & \text { if } \rho=2 \alpha-1<1, \sum_{j<k}\left|q_{j k}\right|>0, \\ O\left(v^{-1}\right), & \text { if } \rho=1, \sum_{j<k}\left|q_{j k}\right|=0,\end{cases}
$$

Similarly, by Lemmata 2.3, 2.4, 2.5 and 2.7, if $V_{j k}^{l} \neq 0$ for some $1 \leq j<k \leq N$ :

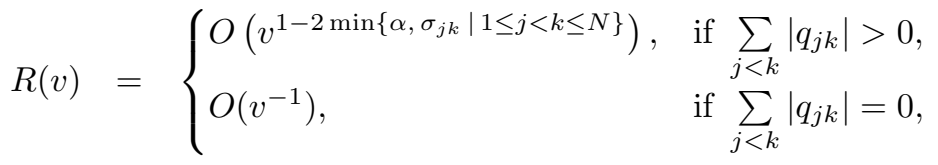

$$
\begin{aligned}
& = \begin{cases}o\left(v^{-\rho}\right), & \text { if } 0 \leq \rho<2 \min \left\{\alpha, \sigma_{j k} \mid 1 \leq j<k \leq N\right\}-1, \\
O\left(v^{-\rho}\right), & \text { if } \rho=2 \min \left\{\alpha, \sigma_{j k} \mid 1 \leq j<k \leq N\right\}-1<1, \\
O\left(v^{-1}\right), & \text { if } \rho=1, \sum_{j<k}\left|q_{j k}\right|=0 .\end{cases}
\end{aligned}
$$

Now, let us compute the following: $\lim _{v \rightarrow \infty} v\left(i\left[S^{D}, \mathbf{p}_{l}\right] \Phi_{\mathbf{v}}, \Psi_{\mathbf{v}}\right)$, using (2.46):

$$
\lim _{v \rightarrow \infty} I_{G, v}=\exp \left(-i \sum_{j<k}^{E} \lim _{v \rightarrow \infty} \int_{-\infty}^{\infty} d s V_{j k}^{s}\left(\mathbf{v}_{j k} s+\mathbf{e}_{1} q_{j k} E s^{2} /\left(2 \mu_{j k}\right)\right)\right)=1 .
$$

We have used the Lebesgue dominated convergence theorem: There exist $0<\delta_{1}, \delta_{2} \leq 1$ such that $\left|\mathbf{v}_{j k} s+\mathbf{e}_{1} q_{j k} E s^{2} /\left(2 \mu_{j k}\right)\right| \geq \sqrt{\delta_{1}\left|\mathbf{v}_{j k} t\right|^{2}+\delta_{2}\left(q_{j k} E /\left(2 \mu_{j k}\right)\right)^{2} t^{4}}$, since, $\left|\hat{\mathbf{v}}_{j k} \cdot \hat{\mathbf{E}}\right| \leq \delta<1$, by (2.13), when $q_{12}=0$, we can take $\delta_{1}=\delta_{2}=1$, and if $q_{12} \neq 0$, we use $\delta_{1}=\delta_{2}=1-\delta$. We can estimate $V_{j k}^{s}\left(\mathbf{v}_{j k} s+\mathbf{e}_{1} q_{j k} E s^{2} /\left(2 \mu_{j k}\right)\right)$ as follows:

$$
\begin{aligned}
\left|V_{j k}^{s}\left(\mathbf{v}_{j k} s+\mathbf{e}_{1} q_{j k} E s^{2} /\left(2 \mu_{j k}\right)\right)\right| & \leq C\left(1+\left|\mathbf{v}_{j k} s+\mathbf{e}_{1} q_{j k} E s^{2} /\left(2 \mu_{j k}\right)\right|\right)^{-\gamma} \\
& \leq C\left(1+\delta_{1} \mathbf{v}_{j k}^{2} s^{2}+\delta_{2}\left|q_{j k} E /\left(2 \mu_{j k}\right)\right|^{2} s^{4}\right)^{-\gamma / 2} \\
& \leq C\left(1+s^{-2 \gamma}\right) .
\end{aligned}
$$

This last term is integrable in $\mathbb{R}$ because $1 / 2<\gamma \leq 1$.

Note that pointwise in $\tau$,

$$
\begin{aligned}
\lim _{v \rightarrow \infty} l_{v}(\tau)= & \left(V_{12}^{v s}(\mathbf{x}+\tau \hat{\mathbf{v}})\left(\mathbf{p}_{l} \Phi_{12}\right), \Psi_{12}\right)-\left(V_{12}^{v s}(\mathbf{x}+\tau \hat{\mathbf{v}}) \Phi_{12},\left(\mathbf{p}_{l} \Psi_{12}\right)\right) \\
& +i\left(\left(\frac{\partial V_{12}^{s}}{\partial x_{l}}\right)(\mathbf{x}+\tau \hat{\mathbf{v}}) \Phi_{12}, \Psi_{12}\right)+i\left(\left(\frac{\partial V_{12}^{l}}{\partial x_{l}}\right)(\mathbf{x}+\tau \hat{\mathbf{v}}) \Phi_{12}, \Psi_{12}\right) .
\end{aligned}
$$


We want to compute $\lim _{v \rightarrow \infty} I(v)$, by (2.47), (2.58) and the Lebesgue dominated convergence theorem, thus showing the rate of convergence when $\rho=0$ in (2.21):

$$
\begin{aligned}
\lim _{v \rightarrow \infty} I(v)= & \int_{-\infty}^{\infty}\left[\left(V_{12}^{v s}(\mathbf{x}+\tau \hat{\mathbf{v}})\left(\mathbf{p}_{l} \Phi_{12}\right), \Psi_{12}\right)-\left(V_{12}^{v s}(\mathbf{x}+\tau \hat{\mathbf{v}}) \Phi_{12},\left(\mathbf{p}_{l} \Psi_{12}\right)\right)\right. \\
& \left.+i\left(\left(\frac{\partial V_{12}^{s}}{\partial x_{l}}\right)(\mathbf{x}+\tau \hat{\mathbf{v}}) \Phi_{12}, \Psi_{12}\right)+i\left(\left(\frac{\partial V_{12}^{l}}{\partial x_{l}}\right)(\mathbf{x}+\tau \hat{\mathbf{v}}) \Phi_{12}, \Psi_{12}\right)\right] d \tau
\end{aligned}
$$

this means in terms of the $J_{1}, J_{2}, J_{3}, J_{4}$ functions that

$$
\begin{aligned}
\lim _{v \rightarrow \infty} J_{1}(v) & =\int\left(V_{12}^{v s}(\mathbf{x}+\tau \hat{\mathbf{v}})\left(\mathbf{p}_{l} \Phi_{12}\right), \Psi_{12}\right) d \tau, \\
\lim _{v \rightarrow \infty} J_{2}(v) & =\int\left(V_{12}^{v s}(\mathbf{x}+\tau \hat{\mathbf{v}}) \Phi_{12},\left(\mathbf{p}_{l} \Psi_{12}\right)\right) d \tau, \\
\lim _{v \rightarrow \infty} J_{3}(v) & =\int\left(\left(\partial V_{12}^{E, s} / \partial x_{l}\right)(\mathbf{x}+\tau \hat{\mathbf{v}}) \Phi_{12}, \Psi_{12}\right) d \tau, \\
\lim _{v \rightarrow \infty} J_{4}(v) & =\int\left(\left(\partial V_{12}^{l} / \partial x_{l}\right)(\mathbf{x}+\tau \hat{\mathbf{v}}) \Phi_{12}, \Psi_{12}\right) d \tau .
\end{aligned}
$$

To justify the use of the Lebesgue dominated convergence theorem observe that $\frac{\partial V_{12}^{s}}{\partial x_{l}}$ and $\frac{\partial V_{12}^{l}}{\partial x_{l}}$ are very short-range. By (2.48) and Lemma 2.3,

$$
\begin{aligned}
& \left|l_{v}(\tau)\right| \leq C\left\|V_{12}^{v s}(\mathbf{x}) e^{-i(\tau / v) H_{0}} \tilde{U}_{D}(\tau / v) \prod_{j^{\prime}<k^{\prime}} f_{j^{\prime} k^{\prime}}\left(\mathbf{p}_{j^{\prime} k^{\prime}}-\mu_{j^{\prime} k^{\prime}} \mathbf{v}_{j^{\prime} k^{\prime}}\right)\left(1+|\mathbf{x}|^{2}\right)^{-2}\right\| \\
& +C\left\|\frac{\partial V_{12}^{s}}{\partial x_{l}}(\mathbf{x}) e^{-i(\tau / v) H_{0}} \tilde{U}_{D}(\tau / v) \prod_{j^{\prime}<k^{\prime}} f_{j^{\prime} k^{\prime}}\left(\mathbf{p}_{j^{\prime} k^{\prime}}-\mu_{j^{\prime} k^{\prime}} \mathbf{v}_{j^{\prime} k^{\prime}}\right)\left(1+|\mathbf{x}|^{2}\right)^{-2}\right\| \\
& +C\left\|\frac{\partial V_{12}^{l}}{\partial x_{l}}(\mathbf{x}) e^{-i(\tau / v) H_{0}} \tilde{U}_{D}(\tau / v) \prod_{j^{\prime}<k^{\prime}} f_{j^{\prime} k^{\prime}}\left(\mathbf{p}_{j^{\prime} k^{\prime}}-\mu_{j^{\prime} k^{\prime}} \mathbf{v}_{j^{\prime} k^{\prime}}\right)\left(1+|\mathbf{x}|^{2}\right)^{-2}\right\| \\
& \leq C h_{12}(|\tau|),
\end{aligned}
$$

where $h_{12} \in L^{1}((0, \infty))$.

Let us find the rate of convergence of (2.59) when $\rho>0$ in (2.21). We estimate the rate of convergence of, $J_{1}$, the first term in the right-hand side of (2.51) (i.e. (2.52) ) to its limit. From (2.52) and (2.60) we have:

$$
\begin{gathered}
J_{1}(v)-\lim _{v \rightarrow \infty} J_{1}(v)=\int_{-\infty}^{-\infty} d \tau\left(V_{12}^{v s}\left(\mathbf{x}+\tau \hat{\mathbf{v}}+\mathbf{e}_{1} q_{12} E \tau^{2} /\left(2 v^{2} \mu_{12}\right)\right) e^{-i \tau \mathbf{p}^{2} /\left(2 v \mu_{12}\right)} \tilde{U}_{D}(\mathbf{v}, \tau / v)\left(\mathbf{p}_{l} \Phi_{0}\right),\right. \\
\left.e^{-i \tau \mathbf{p}^{2} /\left(2 v \mu_{12}\right)} \tilde{U}_{D}(\mathbf{v}, \tau / v) \Psi_{0}\right)-\int_{-\infty}^{-\infty} d \tau\left(V_{12}^{v s}(\mathbf{x}+\tau \hat{\mathbf{v}})\left(\mathbf{p}_{l} \Phi_{12}\right), \Psi_{12}\right) \\
=\int d \tau\left[\left(V_{12}^{v s}(\mathbf{x}+\tau \hat{\mathbf{v}}) e^{-i \mathbf{p} \cdot \mathbf{e}_{1} q_{12} E \tau^{2} /\left(2 v^{2} \mu_{12}\right)} e^{-i \tau \mathbf{p}^{2} /\left(2 v \mu_{12}\right)} \tilde{U}_{D}(\mathbf{v}, \tau / v)\left(\mathbf{p}_{l} \Phi_{0}\right),\right.\right. \\
\left.\quad e^{-i \mathbf{p} \cdot \mathbf{e}_{1} q_{12} E \tau^{2} /\left(2 v^{2} \mu_{12}\right)} e^{-i \tau \mathbf{p}^{2} /\left(2 v \mu_{12}\right)} \tilde{U}_{D}(\mathbf{v}, \tau / v) \Psi_{0}\right) \\
\left.-\left(V_{12}^{v s}(\mathbf{x}+\tau \hat{\mathbf{v}}) e^{-i \mathbf{p} \cdot \mathbf{e}_{1} q_{12} E \tau^{2} /\left(2 v^{2} \mu_{12}\right)} e^{-i \tau \mathbf{p}^{2} /\left(2 v \mu_{12}\right)} \tilde{U}_{D}(\mathbf{v}, \tau / v)\left(\mathbf{p}_{l} \Phi_{0}\right), \Psi_{0}\right)\right] \\
+\int d \tau\left[-\left(\left(\mathbf{p}_{l} \Phi_{0}\right), V_{12}^{v s}(\mathbf{x}+\tau \hat{\mathbf{v}}) \Psi_{0}\right)\right. \\
\left.+\left(e^{-i \mathbf{p} \cdot \mathbf{e}_{1} q_{12} E \tau^{2} /\left(2 v^{2} \mu_{12}\right)} e^{-i \tau \mathbf{p}^{2} /\left(2 v \mu_{12}\right)} \tilde{U}_{D}(\mathbf{v}, \tau / v)\left(\mathbf{p}_{l} \Phi_{0}\right), V_{12}^{v s}(\mathbf{x}+\tau \hat{\mathbf{v}}) \Psi_{0}\right)\right]
\end{gathered}
$$


The latter calculations suggest us to define:

$$
\begin{aligned}
h_{\mathbf{v}}^{(1)}= & \left(V_{12}^{v s}(\mathbf{x}+\tau \hat{\mathbf{v}}) e^{-i \mathbf{p} \cdot \mathbf{e}_{1} q_{12} E \tau^{2} /\left(2 v^{2} \mu_{12}\right)} e^{-i \tau \mathbf{p}^{2} /\left(2 v \mu_{12}\right)} \tilde{U}_{D}(\mathbf{v}, \tau / v)\left(\mathbf{p}_{l} \Phi_{0}\right),\right. \\
& \left.\left(e^{-i \mathbf{p} \cdot \mathbf{e}_{1} q_{12} E \tau^{2} /\left(2 v^{2} \mu_{12}\right)} e^{-i \tau \mathbf{p}^{2} /\left(2 v \mu_{12}\right)} \tilde{U}_{D}(\mathbf{v}, \tau / v)-I\right) \Psi_{0}\right), \\
h_{\mathbf{v}}^{(2)}= & \left(\left(e^{-i \mathbf{p} \cdot \mathbf{e}_{1} q_{12} E \tau^{2} /\left(2 v^{2} \mu_{12}\right)} e^{-i \tau \mathbf{p}^{2} /\left(2 v \mu_{12}\right)} \tilde{U}_{D}(\mathbf{v}, \tau / v)-I\right)\left(\mathbf{p}_{l} \Phi_{0}\right), V_{12}^{v s}(\mathbf{x}+\tau \hat{\mathbf{v}}) \Psi_{0}\right) .
\end{aligned}
$$

With this notation

$$
J_{1}(v)-\lim _{v \rightarrow \infty} J_{1}(v)=\int d \tau\left(h_{\mathbf{v}}^{(1)}+h_{\mathbf{v}}^{(2)}\right)
$$

Let us analyze the rate of convergence of $h_{\mathbf{v}}^{(1)}$. On one hand, with $t=\tau / v$ :

$$
\left\|\left(e^{-i \mathbf{p}_{1} q_{12} E \tau^{2} /\left(2 \mu_{12} v^{2}\right)} e^{-i \mathbf{p}^{2} \tau /\left(2 \mu_{12} v\right)} \tilde{U}_{D}(\mathbf{v}, \tau / v)-I\right) \Psi_{0}\right\|^{2} \leq[C|\tau / v|(1+|\tau / v|)]^{2},
$$

On the other hand:

$$
\left\|\left(e^{-i \mathbf{p}_{1} q_{12} E \tau^{2} /\left(2 \mu_{12} v^{2}\right)} e^{-i \mathbf{p}^{2} \tau /\left(2 \mu_{12} v\right)} \tilde{U}_{D}(\mathbf{v}, \tau / v)-I\right) \Psi_{0}\right\| \leq 2\left\|\Psi_{12}\right\| .
$$

Consider two cases, with $0<a \leq 1$ in mind:

(a) $|\tau / v|<1$ : Clearly we have that $|\tau / v|^{a} \geq|\tau / v|$, therefore:

$$
\left\|\left(e^{-i \mathbf{p}_{1} q_{12} E \tau^{2} /\left(2 \mu_{12} v^{2}\right)} e^{-i \mathbf{p}^{2} \tau /\left(2 \mu_{12} v\right)} \tilde{U}_{D}(\mathbf{v}, \tau / v)-I\right) \Psi_{0}\right\| \leq C|\tau / v|(1+|\tau / v|) \leq C|\tau / v|^{a} .
$$

(b) $|\tau / v| \geq 1$ : In this case $|\tau / v|^{a} \geq 1$, thus:

$$
\left\|\left(e^{-i \mathbf{p}_{1} q_{12} E \tau^{2} /\left(2 \mu_{12} v^{2}\right)} e^{-i \mathbf{p}^{2} \tau /\left(2 \mu_{12} v\right)} \tilde{U}_{D}(\mathbf{v}, \tau / v)-I\right) \Psi_{0}\right\| \leq C \leq C|\tau / v|^{a}
$$

Now we study $\left|h_{\mathbf{v}}^{(1)}(\tau)\right|$ 's decay as $v \rightarrow \infty$ applying Lemma 2.3, and (2.67), (2.68) with $a=\rho$ :

$$
\begin{aligned}
& \left|h_{\mathbf{v}}^{(1)}(\tau)\right| \leq C|\tau / v|^{\rho} \| V_{12}^{v s}\left(\mathbf{x}+\tau \mathbf{p} /\left(\mu_{12} v\right)+\mathbf{e}_{1} q_{12} E \tau^{2} /\left(2 v^{2} \mu_{12}\right)\right) \tilde{U}_{D}(\tau / v) \\
& \times \prod_{j^{\prime}<k^{\prime}} f_{j^{\prime} k^{\prime}}\left(\mathbf{p}_{j^{\prime} k^{\prime}}-\mu_{j^{\prime} k^{\prime}} \mathbf{v}_{j^{\prime} k^{\prime}}\right)\left(1+|\mathbf{x}|^{2}\right)^{-2} \| \text {. }
\end{aligned}
$$

Then

$$
v^{\rho}\left|h_{\mathbf{v}}^{(1)}(\tau)\right| \leq C|\tau|^{\rho} h_{12}(|\tau|) \in L^{1}(-\infty, \infty)
$$

Hence, for $\rho=1$

$$
v \int\left|h_{\mathbf{v}}^{(1)}(\tau)\right| d \tau \leq C .
$$

For $0 \leq \rho<1$, by Lebesgue dominated convergence theorem

$$
\lim _{v \rightarrow \infty} v^{\rho} \int h_{\mathbf{v}}^{(1)}(\tau) d \tau=\int \lim _{v \rightarrow \infty} v^{\rho} h_{\mathbf{v}}^{(1)}(\tau) d \tau=0,
$$

where we used that $\lim _{v \rightarrow \infty} v^{\rho} h_{\mathbf{v}}^{(1)}(\tau)=0$, since by (2.67) and (2.68) with $a=1$ we have $v^{\rho}\left|h_{\mathbf{v}}^{(1)}(\tau)\right| \leq$ $C|\tau| v^{\rho-1}$. 
As a result

$$
\int_{-\infty}^{+\infty} d \tau h_{\mathbf{v}}^{(1)}(\tau)= \begin{cases}o\left(v^{-\rho}\right), & \text { if } 0 \leq \rho<1, \\ O\left(v^{-1}\right), & \text { if } \rho=1\end{cases}
$$

At this moment, we turn our attention to the rate of convergence of $h_{\mathbf{v}}^{(2)}$. When $|\mathbf{x}+\tau \hat{\mathbf{v}}| \leq|\tau| / 2$, we have $|\mathbf{x}| \geq|\tau|-|\mathbf{x}+\tau \hat{\mathbf{v}}| \geq|\tau| / 2$. With the last inequality we can estimate the second factor in the scalar product of (2.65). Let $g$ be a $C_{0}^{\infty}\left(\mathbb{R}^{n}\right)$ such that $g(\mathbf{p}) \hat{\psi}_{12}=\hat{\psi}_{12}$. By (2.67) and (2.68):

$$
\begin{aligned}
v^{\rho} \int_{-\infty}^{\infty} d \tau\left|h_{\mathbf{v}}^{(2)}(\tau)\right| \leq & C \int_{-\infty}^{+\infty} d \tau|\tau|^{\rho}\left(\left\|V_{12}^{v s}(\mathbf{x}+\hat{\mathbf{v}} \tau) g(\mathbf{p}) F(|\mathbf{x}+\hat{\mathbf{v}} \tau| \geq|\tau| / 2)\right\|\right. \\
& \left.+\left\|V_{12}^{v s}(\mathbf{x}+\hat{\mathbf{v}} \tau) g(\mathbf{p})\right\|\left\|F(|\mathbf{x}| \geq|\tau| / 2) \Psi_{12}\right\|\right) .
\end{aligned}
$$

Due to the short-range condition (2.21), the first integral in (2.71) is finite; the fast decay in configuration space of $\Psi_{12}$ makes the second integral in (2.71) be bounded:

$$
\int_{-\infty}^{\infty} d \tau|\tau|^{\rho}\left\|F(|\mathbf{x}| \geq|\tau| / 2) \Psi_{12}\right\|=\int_{-\infty}^{\infty} d \tau|\tau|^{\rho}(1+|\tau|)^{-3}\left\|(1+|\tau|)^{3} F\left(|\mathbf{x}| \geq \frac{|\tau|}{2}\right) \Psi_{12}\right\|<\infty .
$$

Hence, for $\rho=1$

$$
v \int\left|h_{\mathbf{v}}^{(2)}(\tau)\right| d \tau \leq C,
$$

and for $0 \leq \rho<1$, by Lebesgue dominated convergence theorem

$$
\lim _{v \rightarrow \infty} v^{\rho} \int h_{\mathbf{v}}^{(2)}(\tau) d \tau=\int \lim _{v \rightarrow \infty} v^{\rho} h_{\mathbf{v}}^{(2)}(\tau) d \tau=0,
$$

where we used that $\lim _{v \rightarrow \infty} v^{\rho} h_{\mathbf{v}}^{(2)}(\tau)=0$, since by (2.67) and (2.68) with $a=1$ we have $v^{\rho}\left|h_{\mathbf{v}}^{(2)}(\tau)\right| \leq$ $C|\tau| v^{\rho-1}$.

As a result

$$
\int_{-\infty}^{+\infty} d \tau h_{\mathbf{v}}^{(2)}(\tau)= \begin{cases}o\left(v^{-\rho}\right), & \text { if } 0 \leq \rho<1, \\ O\left(v^{-1}\right), & \text { if } \rho=1 .\end{cases}
$$

We have just estimated the rate of convergence of $J_{1}$, the first term in the right-hand side of (2.51). Since $\hat{\phi}_{12} \in C_{0}^{\infty}\left(B_{\mu_{12} \eta}\right)$, we have that $\left(p_{l} \hat{\phi}_{12}\right) \in C_{0}^{\infty}\left(B_{\mu_{12} \eta}\right)$, therefore, we can apply the same treatment to $J_{2}$, the second term in the right-hand side of (2.51). For $J_{3}$, in the right-hand side of (2.51), when we estimate the term with $\partial / \partial x_{l} V_{12}^{E, s}$ we have that

$$
(1+|x|)^{\rho_{s}}\left|\partial / \partial x_{l} V_{12}^{E, s}(x)\right| \leq C(1+|x|)^{-1-\alpha+\rho_{s}}
$$

satisfies the very short-range condition if $\rho_{s}<\alpha \leq 1$. Nevertheless, when $q_{12} \neq 0$, we do not have an extra error term of the form $o\left(v^{-\rho_{s}}\right)$ because in (2.56) and (2.57) $\rho<\alpha$, for that reason one can always choose $\rho_{s}$ such that $\rho<\rho_{s}<\alpha \leq 1$. Regarding $J_{4}$ in (2.51), we estimate the term with $\partial / \partial x_{l} V_{12}^{l}$, one sees that

$$
(1+|x|)^{\rho_{l}}\left|\partial / \partial x_{l} V_{12}^{l}(x)\right| \leq C \begin{cases}(1+|x|)^{-\gamma_{D}-\mu+\rho_{l},} & \text { if } q_{12} \neq 0, \\ (1+|x|)^{-\gamma_{1}+\rho_{l}}, & \text { if } q_{12}=0,\end{cases}
$$

satisfies the very short-range condition if $\rho_{l}<\left\{\begin{array}{ll}\gamma_{D}+\mu-1, & \text { if } q_{12} \neq 0, \\ \gamma_{1}-1, & \text { if } q_{12}=0 .\end{array}\right.$ Therefore, we have another error term of the form

$$
o\left(v^{-\rho_{l}}\right) .
$$


Moreover, when there is at least one pair with non-zero relative charge, we have to estimate the following error, see (2.39) and (2.46). In this case, $\rho<1$, and $-(2 \gamma-1) \leq-(2 \alpha-1) \leq-\rho$, where $\gamma$ is as in Definition 1.2. By equation (2.13):

$$
\begin{aligned}
\left|I_{G, v}-1\right| & \leq \sum_{j<k}^{E} \int_{-\infty}^{\infty} d s\left|V_{j k}^{s}\left(\mathbf{v}_{j k} s+\mathbf{e}_{1} q_{j k} E s^{2} /\left(2 \mu_{j k}\right)\right)\right| \leq C \begin{cases}v^{-(2 \gamma-1)}, & \text { if } 1 / 2<\gamma<1, \\
\frac{\ln v}{v}, & \text { if } \gamma=1,\end{cases} \\
& = \begin{cases}o\left(v^{-\rho}\right), & \text { if } 0 \leq \rho<2 \min \left\{\alpha, \sigma_{j k} \mid 1 \leq j<k \leq N\right\}-1, \\
O\left(v^{-\rho}\right), & \text { if } \rho=2 \min \left\{\alpha, \sigma_{j k} \mid 1 \leq j<k \leq N\right\}-1<1 .\end{cases}
\end{aligned}
$$

Finally, to prove the convergence rate given in (2.44) we sum the terms, corresponding to $I(v), R(v)$, $I_{G, v}$, respectively, in (2.46), recalling (2.56), (2.57), (2.66), (2.70), (2.72), (2.74) and taking in consideration (2.73) with the highest possible values of $\rho_{l}$ in order to have the optimal error rate in all the cases enounced in Theorem 2.8 .

The following reconstruction formula is of independent interest.

THEOREM 2.10. Assume the same hypothesis as in Theorem 2.8, Then

$$
\begin{aligned}
& v\left(i\left[S^{D}-I_{G, v}\right] \Phi_{\mathbf{v}}, \Psi_{\mathbf{v}}\right)-I_{G, v} \int_{-\infty}^{\infty} v d t\left(\left(V_{12}^{s}(\mathbf{x})-V_{12}^{s}\left(\mathbf{v} t+\mathbf{e}_{1} q_{12} E t^{2} /\left(2 \mu_{12}\right)\right)+V_{12}^{l}(\mathbf{x})\right.\right. \\
& \left.\left.-V_{12}^{l}\left(t \mathbf{p} / \mu_{12}-\mathbf{e}_{1} q_{12} E t^{2} /\left(2 \mu_{12}\right)\right)\right) e^{-i t H_{0}} \tilde{U}_{D}(t) \Phi_{\mathbf{v}}, e^{-i t H_{0}} \tilde{U}_{D}(t) \Psi_{\mathbf{v}}\right)=\int_{-\infty}^{\infty} d \tau\left(V_{12}^{E, v s}(\mathbf{x}+\tau \hat{\mathbf{v}}) \Phi_{12}, \Psi_{12}\right) \\
& +\left\{\begin{array}{l}
o\left(v^{-\rho}\right), \quad \text { if } 0 \leq \rho<2 \min \left\{\alpha, \sigma_{j k} \mid 1 \leq j<k \leq N\right\}-1, \\
O\left(v^{-\rho}\right), \quad \text { if } \rho=2 \min \left\{\alpha, \sigma_{j k} \mid 1 \leq j<k \leq N\right\}-1<1, \\
O\left(v^{-1}\right), \quad \text { if } \rho=1, \text { and } \sum\left|q_{j k}\right|=0 .
\end{array}\right.
\end{aligned}
$$

Proof. The left hand side of (2.75) can be written as equal to the right hand side of (2.46) exactly with the same $I_{G, v}$ but with

$$
I(v)=v \int_{-\infty}^{+\infty} d t\left(V_{12}^{v s}(\mathbf{x}) e^{-i t H_{0}} \tilde{U}_{D}(t) \Phi_{\mathbf{v}}, e^{-i t H_{0}} \tilde{U}_{D}(t) \Psi_{\mathbf{v}}\right)
$$

and, with the same $V_{3, t}$ and $V_{t}$ as in the proof of Theorem 2.8

$$
\begin{aligned}
R(v) / v=\int_{-\infty}^{+\infty} d t\left[\left(V_{3, t} e^{-i t H_{0}} \tilde{U}_{D}(t) \Phi_{\mathbf{v}}, e^{-i t H_{0}} \tilde{U}_{D}(t) \Psi_{\mathbf{v}}\right)\right. \\
\left.+\left(\left(e^{-i H t} \Omega_{-}^{D, G, v}-U^{D, G, v}(t)\right) \Phi_{\mathbf{v}}, V_{t} U^{D, G, v}(t) \Psi_{\mathbf{v}}\right)\right] .
\end{aligned}
$$

The convergence rate of $I(v)$ is computed like that one of $J_{1}$, see equations (2.52), (2.64), (2.65), (2.70), (2.72). $R(v)$ and $I_{G, v}$ are estimated like in (2.57) and (2.74), respectively.

\section{Proof of Theorem 1.3;}

Let us consider the states $\Phi \sim \hat{\phi}_{12}(p) \hat{\phi}_{3}\left(p_{3}, \ldots, p_{N}\right), \Psi \sim \hat{\psi}_{12}(p) \hat{\phi}_{3}\left(p_{3}, \ldots, p_{N}\right)$, such that $\hat{\phi}_{12}, \hat{\psi}_{12} \in$ $C_{0}^{\infty}\left(\mathbb{R}^{n}\right)$ and $\hat{\phi}_{3}$ is like in (1.12). Let $\mathbf{y}$ be an element of a two dimensional subspace of $\mathbb{R}^{n}$, for instance, we associate each $\mathbf{y}=\left(y_{1}, y_{2}\right) \in \mathbb{R}^{2}$ with the vector $y_{1} \mathbf{e}_{1}+y_{2} \mathbf{e}_{2} \in \mathbb{R}^{n}$. We express by

$$
\Phi^{\mathbf{y}}=e^{-i \mathbf{p} \cdot \mathbf{y}} \Phi \Leftrightarrow \phi^{\mathbf{y}}=\phi_{12}(\mathbf{x}-\mathbf{y}) \phi_{3}\left(\mathbf{x}_{3}, \ldots, \mathbf{x}_{N}\right), \quad \Psi^{\mathbf{y}}=e^{-i \mathbf{p} \cdot \mathbf{y}} \Psi \Leftrightarrow \psi^{\mathbf{y}}=\psi_{12}(\mathbf{x}-\mathbf{y}) \phi_{3}\left(\mathbf{x}_{3}, \ldots, \mathbf{x}_{N}\right)
$$

the states, translated in the configuration space by $\mathbf{y}$, considered as an vector in $\mathbb{R}^{n}$.

Suppose that $V^{i}=V^{V S, i}+V^{S, i}+V^{L, i} \in \mathcal{V}_{V S R}+\mathcal{V}_{S R}+\mathcal{V}_{L R}, i=1,2$, and that $S^{D}\left(V^{L, 1} ; V^{V S, 1}+V^{S, 1}\right)=$ $S^{D}\left(V^{L, 2} ; V^{V S, 2}+V^{S, 2}\right)$. Then, we can write the potentials $V^{i}, i=1,2$,

$$
V^{i}=\sum_{1 \leq j<k \leq N} V_{j k}^{i}\left(\tilde{\mathbf{x}}_{k}-\tilde{\mathbf{x}}_{j}\right), \quad V_{j k}^{i}=V_{j k}^{v s, i}+V_{j k}^{s, i}+V_{j k}^{l, i}
$$


with, for all $1 \leq j<k \leq N, V_{j k}^{v s, i} \in \mathcal{V}_{V S R}, V_{j k}^{s, i} \in \mathcal{V}_{S R}$, and $V_{j k}^{l, i} \in \mathcal{V}_{L R}$.

It is enough to prove uniqueness for the pair $(1,2)$. Let us assume $q_{12} \neq 0$, the other case is similar and simpler. Note that as $q_{12} \neq 0, V_{12}^{v s, i} \in \mathcal{V}_{E, v s}, V_{12}^{s, i} \in \mathcal{V}_{E, s}$, and $V_{12}^{l, i} \in \mathcal{V}_{E, l}$. We define

$$
\left\{\begin{array}{l}
Q_{12}^{v s}(\mathbf{x})=V_{12}^{v s, 2}(\mathbf{x})-V_{12}^{v s, 1}(\mathbf{x}) \\
Q_{12}^{s}(\mathbf{x})=V_{12}^{s, 2}(\mathbf{x})-V_{12}^{s, 1}(\mathbf{x}) \\
Q_{12}^{l}(\mathbf{x})=V_{12}^{l, 2}(\mathbf{x})-V_{12}^{l, 1}(\mathbf{x}) \\
Q_{12}(\mathbf{x})=Q_{12}^{v s}(\mathbf{x})+Q_{12}^{s}(\mathbf{x})+Q_{12}^{l}(\mathbf{x})
\end{array}\right.
$$

With $\Phi^{\mathbf{y}}$ and $\Psi^{\mathbf{y}}$ as in (2.76), and $\mathbf{p}_{1}=\mathbf{p} \cdot \mathbf{e}_{1}$, the function $f: \mathbb{R}^{2} \rightarrow \mathbb{C}$ is defined as

$$
f(\mathbf{y}):=f_{1}(\mathbf{y})+f_{2}(\mathbf{y})+f_{3}(\mathbf{y})
$$

where

$$
\begin{aligned}
& f_{1}(\mathbf{y}):=\left(Q_{12}^{v s}(\mathbf{x}) \mathbf{p}_{1} \Phi^{\mathbf{y}}, \Psi^{\mathbf{y}}\right) \\
& f_{2}(\mathbf{y}):=-\left(Q_{12}^{v s}(\mathbf{x}) \Phi^{\mathbf{y}}, \mathbf{p}_{1} \Psi^{\mathbf{y}}\right) \\
& f_{3}(\mathbf{y}):=i\left(\left(\frac{\partial Q_{12}^{s}}{\partial x_{1}}+\frac{\partial Q_{12}^{l}}{\partial x_{1}}\right)(\mathbf{x}) \Phi^{\mathbf{y}}, \Psi^{\mathbf{y}}\right) .
\end{aligned}
$$

Let us focus on $f_{1}$. Let $g_{1}$ be a $C_{0}^{\infty}\left(\mathbb{R}^{n}\right)$ function such that $g_{1}(p) \hat{\phi}_{12}(p)=\hat{\phi}_{12}(p)$,

$$
\begin{aligned}
\left|f_{1}(\mathbf{y})\right| \leq & C\left\|Q_{12}^{v s}(\mathbf{x}) g_{1}(\mathbf{p})\right\| \\
\left|f_{1}(\mathbf{y})\right| \leq & C\left(\left\|Q_{12}^{v s}(\mathbf{x}) g_{1}(\mathbf{p}) F(|\mathbf{x}| \geq|\mathbf{y}| / 2)\right\|\right. \\
& \left.+\left\|Q_{12}^{v s}(\mathbf{x}) \mathbf{p}_{1} g_{1}(\mathbf{p})\right\|\left\|F(|\mathbf{x}|<|\mathbf{y}| / 2) \phi_{12}(\mathbf{x}-\mathbf{y})\right\|\right)
\end{aligned}
$$

Inequality (2.79) shows that $f_{1}$ is bounded. By the very short range condition (2.21):

$$
\left\|Q_{12}^{v s}(\mathbf{x}) g_{1}(\mathbf{p}) F(|\mathbf{x}| \geq|\mathbf{y}| / 2)\right\| \in L^{2}\left(\mathbb{R}^{2}\right)
$$

Additionally

$$
\begin{aligned}
\left\|F(|\mathbf{x}|<|\mathbf{y}| / 2) \phi_{12}(\mathbf{x}-\mathbf{y})\right\| & =\left\|\frac{1}{1+|\mathbf{x}-\mathbf{y}|^{2}} F(|\mathbf{x}|<|\mathbf{y}| / 2)\left(1+|\mathbf{x}-\mathbf{y}|^{2}\right) \phi_{12}(\mathbf{x}-\mathbf{y})\right\| \\
& \leq \frac{C}{(1+|\mathbf{y}| / 2)^{2}} \in L^{2}\left(\mathbb{R}^{2}\right) .
\end{aligned}
$$

Then, $f_{1}(\mathbf{y}) \in L^{2}\left(\mathbb{R}^{2}\right)$. Moreover, $f_{1}(\mathbf{y})$ is continuous because the operator $e^{-i \mathbf{p} \cdot \mathbf{y}}$ is strongly continuous on $L^{2}\left(\mathbb{R}^{2}\right)$.

Working with $f_{2}$ and $f_{3}$ is analogous to the case of $f_{1}$, remarking that (1.28) and (1.29) imply that $\frac{\partial Q_{12}^{s}}{\partial x_{1}}+\frac{\partial Q_{12}^{l}}{\partial x_{1}}$ belongs to our very short-range class $\mathcal{V}_{E, v s}$. Thus $f(\mathbf{y}) \in L^{2}\left(\mathbb{R}^{2}\right)$ and it is a bounded continuous function.

The Radon transform of $f(\mathbf{y})$, for any $\mathbf{v}$ in the $\mathbf{y}$-plane satisfying $|\hat{\mathbf{v}} \cdot \hat{\mathbf{E}}|<1$, is given by

$$
\begin{aligned}
\tilde{f}(\hat{\mathbf{v}} ; \mathbf{y}):= & \int_{-\infty}^{\infty} f(\mathbf{y}+\tau \hat{\mathbf{v}}) d \tau=\int_{-\infty}^{\infty}\left[\left(Q_{12}^{v s}(\mathbf{x}+\tau \hat{\mathbf{v}}) \mathbf{p}_{1} \Phi^{\mathbf{y}}, \Psi^{\mathbf{y}}\right)\right. \\
& -\left(Q_{12}^{v s}(\mathbf{x}+\tau \hat{\mathbf{v}}) \Phi^{\mathbf{y}}, \mathbf{p}_{1} \Psi^{\mathbf{y}}\right) \\
& \left.+i\left(\left(\frac{\partial Q_{12}^{s}}{\partial x_{1}}+\frac{\partial Q_{12}^{l}}{\partial x_{1}}\right)(\mathbf{x}+\tau \hat{\mathbf{v}}) \Phi^{\mathbf{y}}, \Psi^{\mathbf{y}}\right)\right]
\end{aligned}
$$


By Theorem 2.8 applied to the pair $(1,2)$, we have that

$$
\begin{aligned}
\tilde{f}(\hat{\mathbf{v}} ; \mathbf{y})= & \lim _{v \rightarrow \infty}\left[v\left(i\left[S^{D}\left(V^{L, 1} ; V^{V S, 1}+V^{S, 1}\right), \mathbf{p}_{1}\right] \Phi_{\mathbf{v}}^{\mathbf{y}}, \Psi_{\mathbf{v}}^{\mathbf{y}}\right)\right. \\
& \left.\quad-v\left(i\left[S^{D}\left(V^{L, 2} ; V^{V S, 2}+V^{S, 2}\right), \mathbf{p}_{1}\right] \Phi_{\mathbf{v}}^{\mathbf{y}}, \Psi_{\mathbf{v}}^{\mathbf{y}}\right)\right] \\
\equiv & 0 .
\end{aligned}
$$

Then, the Plancherel formula associated with the Radon transform 22] implies that $f(\mathbf{y})=0$. From (2.78) we have that

$$
\frac{\partial}{\partial y_{1}}\left(Q_{12} \Phi^{\mathbf{y}}, \Psi^{\mathbf{y}}\right)=-i f(\mathbf{y})
$$

This implies that $\left(Q_{12} \Phi^{\mathbf{y}}, \Psi^{\mathbf{y}}\right)$ does not depend on $y_{1}$. Moreover, $\lim _{\left|y_{1}\right| \rightarrow \infty}\left(Q_{12} \Phi^{\mathbf{y}}, \Psi^{\mathbf{y}}\right)=0$ by (1.26), (1.27) and (1.29). Therefore, $\left(Q_{12} \Phi^{\mathbf{y}}, \Psi^{\mathbf{y}}\right) \equiv 0$. In particular $\left(Q_{12} \Phi^{0}, \Psi^{0}\right)=\left(Q_{12} \phi_{12}, \psi_{12}\right)=0$, what implies by the density of the states $\phi_{12}, \psi_{12}$ that $Q_{12}(\mathbf{x}) \equiv 0$ a.e. We conclude that the total potential $V$ is uniquely determined by the high-velocity limit of the commutator of any Dollard scattering operator $S^{D}$ and some component of the momentum.

We consider the reconstruction problem of the total potential $V$ as in (1.36), by means of Theorem 2.8 We assume $q_{12} \neq 0$ because the case $q_{12}=0$ is easier. Let us compute $V_{12}:=V_{12}^{v s}+V_{12}^{s}+V_{12}^{l} \in \mathcal{V}_{V S R}+\mathcal{V}_{S R}+\mathcal{V}_{L R}$ from the high-velocity limit of $\left[S^{D}, \mathbf{p}_{1}\right]$. We substitute $Q_{12}^{v s}$ by $V_{12}^{v s}, Q_{12}^{s}$ by $V_{12}^{s}$ and $Q_{12}^{l}$ by $V_{12}^{l}$ in (2.78). We know $\lim _{v \rightarrow \infty} v\left(i\left[S^{D}, \mathbf{p}_{1}\right] \Phi_{\mathbf{v}}^{\mathbf{y}}, \Psi_{\mathbf{v}}^{\mathbf{y}}\right)$ for all $\Phi^{\mathbf{y}}$ and $\Psi^{\mathbf{y}}$ as in (2.76). Then, by Theorem 2.8 and (2.81) we reconstruct $\tilde{f}(\hat{\mathbf{v}} ; \mathbf{y})$ and by the inversion of the Radon transform [22, we uniquely reconstruct $f(\mathbf{y})$. From (1.26), (1.27), (1.28) and (1.29) $f$ is integrable along any line and $\lim _{\mathbf{y} \rightarrow \infty}\left(\left(V_{12}\right) \Phi^{\mathbf{y}}, \Psi^{\mathrm{y}}\right)=0$. Then we have

$$
\left(V_{12} \phi_{12}, \psi_{12}\right)=i \int_{0}^{\infty} f\left(y_{1}, 0\right) d y_{1}
$$

in a dense set in $L^{2}$. Hence $V_{12}$ is obtained almost everywhere as a function. Repeating this process for all pairs we reconstruct $V$.

REMARK 2.11. As we have already mentioned in Remark 1.6 the reconstruction formula (2.75) from Theorem 2.10 is simpler than the formula (2.44) in Theorem 2.8, Let us show how (2.75) can be used. Let us suppose that $q_{12} \neq 0$. The case $q_{12}=0$ follows in the same way. The potentials $V_{12}^{E, v s} \in \mathcal{V}_{E, v s}, V_{12}^{E, s} \in \mathcal{V}_{E, s}$, $V_{12}^{E, l} \in \mathcal{V}_{E, l}$ are the very short-, short- and long-range potentials, respectively, for the pair $(1,2)$. Let us assume that we want to recover $V_{12}^{E, v s}$ knowing $V_{12}^{E, s}, V_{12}^{E, l}$ and the high-velocity limit of $S^{D}$ for each $\Phi^{\mathbf{y}}$ and $\Psi^{\mathrm{y}}$ as in (2.76). Defining

$$
h(\mathbf{y})=\left(V_{12}^{E, v s}(\mathbf{x}) \Phi^{\mathbf{y}}, \Psi^{\mathbf{y}}\right),
$$

using Theorem 2.10 and inverting the Radon transform we obtain $h(\mathbf{y})$. Then, we can compute $\left(V_{12}^{E, v s} \phi_{12}, \psi_{12}\right)$ $=h(0)$ in a dense set in $L^{2}$. This implies that we recover $V_{12}^{E, v s}$ almost everywhere as a function.

\section{Acknowledgment}

We thank Professor Patrick Joly for his kind hospitality at the project POEMS at Institut National de Recherche en Informatique et en Automatique Paris-Rocquencourt, where this work was partially done. We also greatly appreciate the comments made by the referee.

\section{References}

[1] Adachi, T.; Kamada, T; Kazuno, M.; Toratani K. "On multidimensional inverse scattering in an external electric field asymptotically zero in time". Inverse Problems 27 (2011) 065006.

[2] Adachi, T.; Maehara K. "On multidimensional inverse scattering for Stark Hamiltonians". J. Math. Phys. 48 (2007) 042101. 
[3] Adachi, T.; Tamura, H. "Asymptotic completeness for long-range many-particle systems with Stark effect". J. Math. Sci. Univ. Tokyo 2 (1995) 76-116.

[4] Arians, S. "Eine neue Phasenraumlokalisierung im Vollständigkeitsbeweis für Hamiltonoperatoren mit Magnetfeld (A New Phase Space Localization in the Proof of Completeness for Hamiltonians with Magnetic Field), diploma thesis (German only)". RWTH Aachen 1994, Logos Verlag, Berlin (1996).

[5] Arians, S. "Geometric approach to inverse scattering for the Scrödinger equation whith magnetic and electric potentials". J. Math. Phys. 38 (1997) 2761-2773.

[6] Arians, S. "Geometric approach to inverse scattering for hydrogen-like systems in a homogeneous magnetic field". J. Math. Phys. 39 (1998) 1730-1743.

[7] Avron, J. E.; Herbst, I. W. "Spectral and scattering theory of Schrödinger operators related to the Stark effect". Comm. Math. Phys. 52 (1977) 239-254.

[8] Ballesteros, M.; Weder, R. "High-velocity estimates for the scattering operator and Ahanorov-Bohm effect in three dimensions". Commun. Math. Phys. 285 (2008) 345-398.

[9] Ballesteros, M.; Weder, R. "The Aharonov-Bohm effect and Tonomura et al. experiments: Rigorous results". J. Math. Phys. 50 (2009) 122108.

[10] Ballesteros, M.; Weder, R. "Aharonov-Bohm Effect and High-Velocity Estimates of Solutions to the Schödinger Equation". Commun. Math. Phys. 303 (2011) 175-211.

[11] Daudè, T; Nicoleau, F. "Recovering the mass and the charge of a Reissner-Nordström black hole by an inverse scattering experiment". Inverse Problems 24 (2008) 025017. See also Corrigendum: Recovering the mass and the charge of a Reissner-Nordström black hole by an inverse scattering experiment, Inverse Problems 25 (2009) 059801.

[12] Daudè, T; Nicoleau, F. "Inverse scattering in de Sitter-Reissner-Nordström black hole spacetimes". Rev. Math. Phys. 22 (2010) 431-484.

[13] Enss, V. "Asymptotic completeness for quantum-mechanical potential scattering II. Singular and longrange potentials". Ann. Phys. 119 (1979) 117-132.

[14] Enss, V. "Propagation properties of quantum scattering states". J. Funct. Anal. 52 (1983) 219-251.

[15] Enss, V. "A New Look at the Multidimensional Inverse Scattering Problem". Understanding Physics, A.K. Richter ed., Copernicus Gesellschaft, Katlenburg-Lindau (1998) 31-48.

[16] Enss, V.; Jung, W. "Geometrical Approach to Inverse Scattering". Appeared in the proceedings of the First MaPhySto Workshop on Inverse Problems, 22-24 April 1999, Aarhus. MaPhySto Miscellanea (1999) 5-21.

[17] Enss, V.; Weder, R. "Inverse potential scattering: a geometrical approach". Mathematical quantum theory II. Schrödinger operators (Vancouver, BC, 1993) CRM Proc. Lecture Notes, Amer. Math. Soc. Providence, RI 8 (1995) 151-162.

[18] Enss, V; Weder, R. "Uniqueness and reconstruction formulae for inverse N-particle scattering", appeared in "Differential Equations and Mathematical Physics", Proceedings of the International Conference, Univ. of Alabama at Birmingham, March 1994, I. Knowles editor, International Press Boston (ca. 1995) 53-66.

[19] Enss, V; Weder, R. "The geometric approach to multidimensional inverse scattering". J. Math. Phys. 36 (1995) 3902-3921. 
[20] Enss, V; Weder, R. "Inverse two-cluster scattering". Inverse Problems 12 (1996) 409-418.

[21] Graf, G. M. "A remark on long-range Stark scattering". Helv. Phys. Acta 64 (1991) 1167-1174.

[22] Helgason, S. "The Radon transform, 2nd ed., Progress in Mathematics 5, Boston: Birkhäuser (1999).

[23] Herbst, I.; Møller, J. S.; Skibsted, E. "Asymptotic completeness for N-body Stark Hamiltonians". Comm. Math. Phys. 174 (1996) 509-535.

[24] Hörmander, L. "The existence of wave operators in scattering theory". Math. Z. 146 (1976) 69-91.

[25] Ito, Hiroshi T. "Inverse scattering problems for Dirac operator with time-dependent electromagnetic potentials". (Japanese) Spectral-scattering theory and related topics (Japanese) (Kyoto, 1997). Sūrikaisekikenkyūsho Kōkyūroku 1047 (1998) 26-35

[26] Ito, Hiroshi T. "An inverse scattering problem for Dirac equations with time-dependent electromagnetic potentials". Publ. RIMS, Kyoto Univ. 34 (1998) 355-381.

[27] Jung, W. "Der geometrische Ansatz zur inversen Streutheorie bei der Dirac-Gleichung". Diplomarbeit RWTH-Aachen, 1996.

[28] Jung, W. "Geometrical approach to inverse scattering for the Dirac equation". J. Math. Phys. 38 (1997) 39-48.

[29] Jung, W. "Gauge Transformations and Inverse Quantum Scattering with Medium-Range Magnetic Fields". Math. Phys. Electron. J. 11 (2005) paper 5.

[30] Kitada, H; Yajima, K. "A scattering theory for time-dependent long-range potentials". Duke Math. J. 49 (1982) 341-376.

[31] Korotyaev, E. L. "On the scattering theory of several particles in an external electric field". Math. USSR Sb. 60 (1988) 177-196.

[32] Nicoleau, F. "An inverse scattering problem with the Aharonov-Bohm effect". J. Math Phys. 41 (2000) 5223-5237.

[33] Nicoleau, F. "Inverse scattering for Stark Hamiltonians with short-range potentials". Asymptot. Anal. 35 (2003) 349-359.

[34] Nicoleau, F. "An inverse scattering problem for short-range systems in a time-periodic electric field". Math. Res. Lett. 12 (2005) 10001-10012.

[35] Reed, M.; Simon, B. "Vol. III. Scattering Theory". Academic Press, New York (1979).

[36] Sandoval Romero, M.; Weder, R. "The Initial Value Problem, Scattering and Inverse Scattering, for Non-Linear Schrödinger Equations with a Potential and a Non-Local Non-Linearity". J. Phys. A 39 (2006) 11461-11478.

[37] Tamura, H. "Scattering Theory for N-Particle Systems with Stark Effect: Asymptotic Completeness". Publ. RIMS, Kyoto Univ. 29 (1993) 869-884.

[38] Veselić, K.; Weidmann, J. "Potential scattering in a homogeneous electrostatic field". Math. Z. 156 (1977) 93-104.

[39] Weder, R. "Multidimensional inverse scattering in an electric field". J. Funct. Anal. 139 (1996) $441-465$.

[40] Weder, R. "Inverse scattering for $N$-body systems with time-dependent potentials". Inverse problems of wave propagation and diffraction (Aix-les-Bains, 1996), Lecture Notes in Physics 486 (1997) 27-46. 
[41] Weder, R. "Inverse scattering for the nonlinear Schrödinger equation and $L^{p}-L^{p}$ estimates". Spectral and scattering theory and related topics (Japanese) (Kyoto, 1999). Sūrikaisekikenkyūsho Kōkyūroku 1156 (2000) 157-168.

[42] Weder, R. " $L^{p}-L^{\dot{p}}$ estimates for the Schrödinger equation on the line and inverse scattering for the nonlinear Schrödinger equation with a potential". J. Funct. Anal. 170 (2000) 37-68.

[43] Weder, R. "Uniqueness of inverse scattering for the nonlinear Schrödinger equation and reconstruction of the potential and the nonlinearity". Mathematical and numerical aspects of wave propagation (Santiago de Compostela, 2000), SIAM, Philadelphia, PA (2000) 631-634.

[44] Weder, R. "Inverse scattering on the line for the nonlinear Klein-Gordon equation with a potential" . J. Math. Anal. Appl. 252 (2000) 102-123.

[45] Weder, R. "Inverse scattering for the non-linear Schrödinger equation: reconstruction of the potential and the non-linearity". Math. Meth. Appl. Sci. 24 (2001) 245-254.

[46] Weder, R. "Inverse scattering for the nonlinear Schrödinger equation. II. Reconstruction of the potential and the nonlinearity in the multidimensional case". Proc. Amer. Math. Soc. 129 (2001) 3637-3645.

[47] Weder, R. "Direct and inverse scattering for the nonlinear Schrödinger equation with a potential". VI Seminar on Free Boundary Value Problems and their Applications, Part 1 (Spanish) (Rosario, 1998). MAT - Serie A, 3 (2001) 13-20.

[48] Weder, R. "The Aharonov-Bohm effect and time-dependent inverse scattering theory". Inverse Problems 18 (2002) 1041-1056.

[49] Weder, R. "Multidimensional inverse scattering for the nonlinear Klein-Gordon equation with a potential". J. Differential Equations 184 (2002) 62-77.

[50] Weder, R. "The time-dependent approach to inverse scattering theory". Contemporary Mathematics $\mathbf{3 2 7}$ (2003) 359-377.

[51] Weder, R. "The $L^{p}-L^{p^{\prime}}$ estimate for the Schrödinger equation on the half-line". J. Math. Anal. Appl. 281 (2003) 233-243.

[52] Weder, R. "Scattering for the forced non-linear Schrödinger equation with a potential on the half-line". Math. Meth. Appl. Sci. 28 (2005) 1219-1236.

[53] Weder, R. "The forced non-linear Schrödinger equation with a potential on the half-line". Math. Meth. Appl. Sci. 28 (2005) 1237-1255.

[54] Weder, R. " Topological Effects in Quantum Mechanics and High-Velocity Estimates". Int. J. Mod. Phys. D 20 (2011) 951-961.

[55] Weder, R. "The electric Aharonov-Bohm effect". J. Math. Phys. 52 (2011) 052109.

[56] Zorbas, J. "Scattering theory for Stark Hamiltonians involving long-range potentials". J. Math. Phys. 19 (1978) 577-580. 